\title{
Curvature theory of boundary phases: the two-dimensional case
}

\author{
ANDREA BRAIDES ${ }^{\dagger}$ \\ Dipartimento di Matematica, Università di Roma 'Tor Vergata', via della Ricerca Scientifica, \\ 00133 Rome, Italy \\ AND \\ ANDREA MALCHIODI ${ }^{\ddagger}$ \\ Institute for Advanced Study, School of Mathematics, 1 Einstein Drive, Princeton, NJ 08540, \\ USA
}

[Received 7 March 2001 and in revised form 20 June 2002]

\begin{abstract}
We describe the behaviour of minimum problems involving non-convex surface integrals in $2 \mathrm{D}$, singularly perturbed by a curvature term. We show that their limit is described by functionals which take into account energies concentrated on vertices of polygons. Non-locality and non-compactness effects are highlighted.
\end{abstract}

Keywords: Surface energies; curvature functionals; phase transitions; $\Gamma$-convergence; non convex problems

\section{Introduction}

The starting point of this work is the study of minimum problems related to the equilibrium of elastic crystals (see for example, $[15,16]$ for the variational formulation, $[8,9]$ for a derivation of the model from statistical considerations, [3] for its links with Ising systems, and [20,25] for an analogous derivation as a singular perturbation of the Allen-Cahn model). The model problem we have in mind is that of finding sets minimizing a (possibly highly anisotropic) 'perimeter functional' (i.e. a line integral on the boundary, that reduces to the usual perimeter if $\partial E$ is regular and $\psi$ is identically 1 ), of the form

$$
\min \left\{\int_{\partial E} \psi\left(v_{E}\right) \mathrm{d} \mathcal{H}^{1}: E_{0} \subseteq E\right\}
$$

where the minimum is computed among all sets $E \subset \mathbb{R}^{2}$ with boundary of class $C^{1}$ and containing a fixed open set $E_{0}$. Here, $\psi$ is a Borel function, $v_{E}$ denotes the (appropriately oriented) tangent to $E$ and $\mathcal{H}^{1}$ is the one-dimensional (Hausdorff) surface measure. Another model problem is that of local minimizers of the same anisotropic perimeter, related to

$$
\min \left\{\int_{\partial E} \psi\left(v_{E}\right) \mathrm{d} \mathcal{H}^{1}:\left|E_{0} \Delta E\right| \leqslant \delta\right\}
$$

where $\delta>0$ is a fixed constant ( $A \Delta B$ stands for the symmetric difference of the sets $A$ and $B$ ).

\footnotetext{
Corresponding author. Email: braides@mat.uniroma2.it

*Email: malchiod@ias.edu
} 
Problems of the above type, and some of their perturbations, can be tackled following the socalled direct method of the calculus of variations. First, problems (1) and (2) can be 'relaxed' by admitting as competing sets all sets with finite perimeter (see $[5,18])$. Then, if $\psi$ is larger than a fixed constant on $S^{1}$ and if its homogeneous positive extension of degree one outside $S^{1}$ is a convex function, classical results imply that the surface integral in (1) and (2) is lower semicontinuous and coercive in the appropriate topology of the $L^{1}$-convergence of characteristic functions. The application of the direct method of the calculus of variations thus yields the existence of minimizing sets of finite perimeter and, if $\psi^{2}$ is smooth and strictly convex (hypotheses are usually made on $\psi^{2}$ since $\psi$ is positively homogeneous and hence non-convex on radial directions), regularity results for minimal surfaces assure that such minimizers are regular. On the other hand if $\psi^{2}$ is not convex, then the minimum problems (1) or (2) may not possess solutions. It can be seen (see for example, [21]) that the application of the direct method of the calculus of variations gives minimizing sequences with increasingly wiggly boundaries (even though with equi-bounded total area). Their limits can be described (see [4]) as minimizers of a 'relaxed' problem of the same type: in the case of (1), for example,

$$
\min \left\{\int_{\partial E} \bar{\psi}\left(v_{E}\right) \mathrm{d} \mathcal{H}^{1}: E_{0} \subseteq E\right\},
$$

where the new surface energy density $\bar{\psi}$ is simply the convex envelope of the one-homogeneous extension of $\psi$ to $\mathbb{R}^{2}$. This process may lead to non-strictly convex integrands, which in turn may yield non-unique and non-smooth solutions. In this case, it may be necessary to consider higherorder terms in the surface energy to explain solutions with sharp corners and facets (see also [30]; a similar phenomenon is studied in [19]). Note that so far the problem can be framed in an $n$ dimensional framework, upon replacing curves by hypersurfaces.

In this paper we study, in a genuinely two-dimensional setting, the case when we add a singular perturbation by a curvature term in (1) (or analogously in (2)), obtaining a minimum problem of the form

$$
\min \left\{\int_{\partial E}\left(\psi\left(v_{E}\right)+\varepsilon^{2} \kappa^{2}\right) \mathrm{d} \mathcal{H}^{1}: E_{0} \subseteq E\right\},
$$

where now the minimum is taken among sets with $C^{2}$ boundary and $\kappa(x)$ denotes the curvature of $\partial E$ at $x$. In this way, oscillating boundaries are penalized if they introduce large curvatures.

In a way similar to $[22,24,25]$, in order to understand the behaviour of minimizers for (4) we may study the (equivalent) $\varepsilon$-rescaled minimum problem

$$
\min \left\{\int_{\partial E}\left(\frac{\psi\left(v_{E}\right)-\bar{\psi}\left(v_{E}\right)}{\varepsilon}+\varepsilon \kappa^{2}\right) \mathrm{d} \mathcal{H}^{1}: E_{0} \subseteq E\right\} .
$$

We assume for simplicity that $\psi\left(v_{E}\right)=\bar{\psi}\left(v_{E}\right)$ only on a finite number of directions $v_{1} \ldots, v_{N}$ $(N>2)$. One can easily check that under this assumption $\psi$ must satisfy

$$
\psi(v)>\frac{\sin \left(v_{i+1}-v\right)}{\sin \left(v_{i+1}-v_{i}\right)} \psi\left(v_{i}\right)+\frac{\sin \left(v-v_{i}\right)}{\sin \left(v_{i+1}-v_{i}\right)} \psi\left(v_{i+1}\right), \quad \forall v \in\left(v_{i}, v_{i+1}\right), \forall i=1, \ldots, N
$$

(we identify $v_{N+1}$ with $v_{1}$ ). Note that this condition rules out a smooth behaviour near $v_{1}, \ldots, v_{N}$ as in the energies considered in [19]. The problem can then be rewritten as

$$
\min \left\{\int_{\partial E}\left(\frac{\varphi\left(v_{E}\right)}{\varepsilon}+\varepsilon \kappa^{2}\right) \mathrm{d} \mathcal{H}^{1}: E_{0} \subseteq E\right\}
$$

where $\varphi: S^{1} \rightarrow[0,+\infty)$ vanishes only on those preferred directions. 


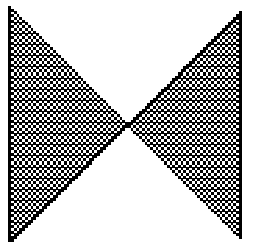

$\mathrm{E}$

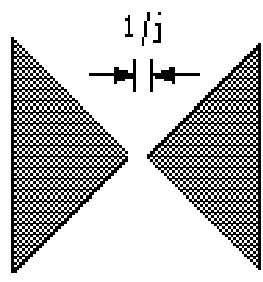

$\mathrm{E}_{\mathrm{j}}$

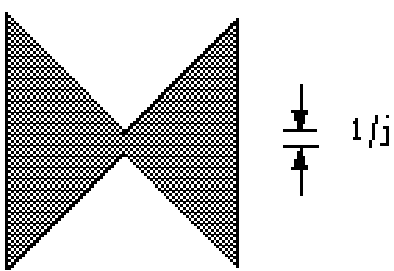

$E_{j}$

FIG. 1. Two approximations with simple vertices.

Our main result is to describe the asymptotic behaviour as $\varepsilon \rightarrow 0$ of problem (6), showing that the minimizers $E_{\varepsilon}$ tend, up to translations, to sets $E$ which in turn minimize a limit energy. This limit energy can be computed by using the techniques of $\Gamma$-convergence (see $[10,11,13])$. We define the functional $F_{\varepsilon}$ on sets of finite perimeter as

$$
F_{\varepsilon}(E)= \begin{cases}\int_{\partial E}\left(\frac{\varphi\left(v_{E}\right)}{\varepsilon}+\varepsilon \kappa^{2}\right) \mathrm{d} \mathcal{H}^{1} & \text { if } E \text { is of class } C^{2} \\ +\infty & \text { otherwise }\end{cases}
$$

and we compute their $\Gamma$-limit $G$ with respect to the $L^{1}$ and $L_{\text {loc }}^{1}$-convergence of characteristic functions. As an example, in the simplest case when $\varphi$ is symmetric with respect to both axes and the preferred directions coincide with the coordinate directions, the domain of the limit $G$ is simply the set of the coordinate polyrectangles and $G(E)=c \#(V(E))$, where $V(E)$ is the set of vertices of the polyrectangle $E$. The constant $c$ can be computed as

$$
c=2 \int_{S} \sqrt{\varphi(s)} \mathrm{d} \mathcal{H}^{1}(s)
$$

where $S$ is the minimal arc in $S^{1}$ connecting $(1,0)$ and $(0,1)$. Hence, the limit problem is trivially

$$
\min \left\{c \#(V(E)): E \text { a coordinate polyrectangle, } E_{0} \subseteq E\right\}
$$

and the minimizers of the limit problem are simply all coordinate rectangles containing $E_{0}$. Note that the limits of minimizers $E_{\varepsilon}$ of (4) minimize both (3) and (9), so that they are coordinate rectangles (since they must minimize the number of vertices) containing $E_{0}$ of minimal perimeter (since the energy in (3) coincides with the Euclidean perimeter on polyrectangles).

In the general case, we show that the domain of the limit energy consists of those polygons whose tangents belong to the set of the preferred directions $\left\{v_{1}, \ldots, v_{N}\right\}$, and that the limit energy $G$ is much more complicated than (9). If $E$ contains only simple vertices (which can be also phrased: if $\partial E$ is locally Lipschitz) we define

$$
F(E)=\sum\left\{g\left(v^{-}(v), v^{+}(v)\right): v \in V(E)\right\},
$$



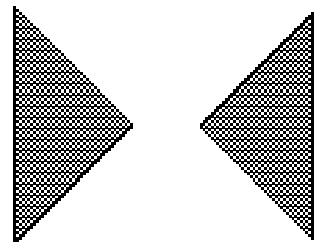

$\mathrm{E}$

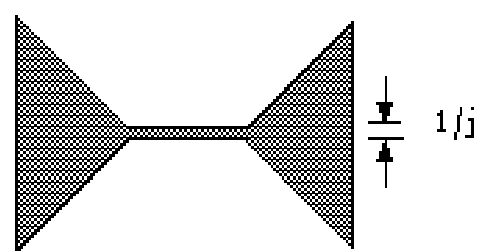

$E_{j}$

FIG. 2. Approximation giving a non-local effect.

where $g$ is given by

$$
g\left(v_{1}, v_{2}\right)=2 \int_{A\left(v_{1}, v_{2}\right)} \sqrt{\varphi(s)} \mathrm{d} \mathcal{H}^{1}(s)
$$

$\left(A\left(v_{1}, v_{2}\right)\right.$ is the minimal arc connecting $v_{1}$ and $v_{2}$ in $\left.S^{1}\right)$ and $v^{ \pm}(v)$ are the two tangents at $v$. If, loosely speaking, $E$ is such that approximating sequences $E_{\varepsilon}$ may be chosen 'uniformly close' to $E$ then we prove that $G(E)=F(E)$. In the general case, the value $G(E)$ is obtained as

$$
G(E)=\inf \left\{\liminf _{j} F\left(E_{j}\right): E_{j} \rightarrow E, E_{j} \text { with simple vertices }\right\} .
$$

This formula hides two types of degenerate behaviours. First of all, we have to take into account that when two or more vertices coincide the set $E$ may be approximated in many different ways and (12) highlights that the approximation of minimal energy must be chosen. Figure 1 shows two different approximations $E_{j}$ with simple vertices of a set $E$ with a 'double' vertex. In addition, the energy $G$ may be non-local: in a sense, a polygon may be completed by adding segments pointing in some of the preferred directions, which must be considered as degenerate parts of $E$; the energy $G(E)$ takes into account the 'minimal' of such completions. In Fig. 2 the corresponding optimal approximation is pictured. This effect is analogous to that highlighted in [6] for functionals depending on the square of the curvature. As a consequence of formula (12), we get that the study of minimizers of problems involving $G$ corresponds to the analysis of minimizing sequences of corresponding problems involving $F$. In particular, we deduce that the limit problem of (6) admits as solutions all the convex polygons with tangents in the preferred directions.

Once the form of the $\Gamma$-limit is computed, we may apply our results also to other problems for which the solution is less immediate, such as

$$
\min \left\{\int_{\partial E}\left(\frac{\varphi\left(v_{E}\right)}{\varepsilon}+\varepsilon \kappa^{2}\right) \mathrm{d} \mathcal{H}^{1}:\left|E_{0} \Delta E\right| \leqslant \delta\right\}
$$

or

$$
\min \left\{\int_{\partial E}\left(\frac{\varphi\left(v_{E}\right)}{\varepsilon}+\varepsilon \kappa^{2}\right) \mathrm{d} \mathcal{H}^{1}+\left|E_{0} \Delta E\right|\right\}
$$

where $E_{0}$ is some fixed set. The latter problem is also of interest in some models in Image Processing where energies depending on curvatures and on (the number of) vertices are considered 
(see $[12,23,27])$. Note that the solution to problem (14) may be given by a set $E$ satisfying $G(E)<F(E)$ (see the example in Section 6.2).

Finally, we note that, since the solutions of the limit problem are polygons with fixed orientations, it is very tempting to link this approximation result to the theory of crystalline growth as recently developed (see $[7,17,28,29]$ ), where non-strictly convex $\psi$ are considered.

The paper is organized as follows. Section 2 contains the statement of the main results in terms of $\Gamma$-convergence and the necessary notation. In Sections 3 and 4 we prove the lower and upper bounds for the limit energy. In Section 5 some cases are dealt with when the limit energy is local: i.e., it can be written as a sum of energies concentrated on vertices. Finally, in Section 6 we consider the pathological case when we do not have a boundedness condition on the perimeter, giving a qualitative description of the shape of sequences with equi-bounded energy. We also give an example where $\Gamma$-limits computed in the $L^{1}$ and $L_{\text {loc }}^{1}$ topology differ.

\section{Main results}

\subsection{Statement of the main results}

For every open set $E \subseteq \mathbb{R}^{2}$ of class $C^{2}$ and every $\varepsilon>0$, we define the energy

$$
F_{\varepsilon}(E)=\int_{\partial E}\left(\frac{1}{\varepsilon} \varphi(v)+\varepsilon \kappa^{2}\right) \mathrm{d} \mathcal{H}^{1}
$$

where $v=v(x)$ is the tangent direction to $\partial E$ in $x$, defined in such a way that $\left(\nu_{2},-v_{1}\right)$ coincides with the outer unit normal to $\partial E$ in $x$. With $\mathcal{H}^{1}$ we denote the one-dimensional Hausdorff measure. The quantity $\kappa=\kappa(x)$ denotes the curvature of $\partial E$ in $x$, and $\varphi: S^{1} \rightarrow[0,+\infty)$ (we identify $S^{1}$ with $\mathbb{R} \bmod 2 \pi$ ) is a continuous function with the following property:

$$
\exists v_{1}, \ldots v_{N} \in S^{1}, \quad v_{1}<v_{2} \cdots<v_{N}<v_{N+1}=v_{1}+2 \pi \quad \text { such that } \varphi^{-1}(0)=\left\{v_{1}, \ldots v_{N}\right\} .
$$

We will always assume that

$$
\left|v_{i}-v_{i+1}\right|<\pi, \quad i=1, \ldots, N .
$$

We will identify sets $E$ with their characteristic function $\chi_{E}$, and then the functional given by formula (15) will be identified with the functional $F_{\varepsilon}: L^{1}\left(\mathbb{R}^{2}\right) \rightarrow[0,+\infty]$ given by

$$
F_{\varepsilon}(u)= \begin{cases}\int_{\partial E}\left(\frac{1}{\varepsilon} \varphi(\nu)+\varepsilon \kappa^{2}\right) \mathrm{d} \mathcal{H}^{1} & \text { if } u=\chi_{E} \text { and } E \text { is of class } C^{2} \\ +\infty & \text { otherwise. }\end{cases}
$$

With an additional slight abuse of notation, we say that a sequence of sets $\left(E_{n}\right) \subseteq \mathbb{R}^{2}$ converges to $E \subseteq \mathbb{R}^{2}$ in $L^{1}\left(\mathbb{R}^{2}\right)$ if $\chi_{E_{n}} \rightarrow \chi_{E}$ in $L^{1}\left(\mathbb{R}^{2}\right)$.

For $\theta_{1}, \theta_{2} \in S^{1}, \theta_{1} \neq \theta_{2}+\pi$, let $A_{\left(\theta_{1}, \theta_{2}\right)}$ denote the shortest of the two $\operatorname{arcs}$ in $S^{1}$ connecting $\theta_{1}$ and $\theta_{2}$. We assume that $A_{\left(\theta_{1}, \theta_{2}\right)}$ is oriented in the direction going from $\theta_{1}$ to $\theta_{2}$. We define $g: S^{1} \times S^{1} \rightarrow[0,+\infty)$ in the following way:

$$
g\left(\theta_{1}, \theta_{2}\right)= \begin{cases}2 \int_{A_{\left(\theta_{1}, \theta_{2}\right)}} \sqrt{\varphi(\nu)} \mathrm{d} \mathcal{H}^{1}(\nu) & \text { if } \theta_{i} \in\left\{v_{1}, \ldots v_{N}\right\} \quad i=1,2 \\ +\infty & \text { otherwise. }\end{cases}
$$


Note that $g\left(\theta_{2}, \theta_{1}\right)=g\left(\theta_{1}, \theta_{2}\right)$.

An admissible polygon is a set $P \subseteq \mathbb{R}^{2}$ whose boundary is a polygonal composed of segments whose directions lie in the set $\left\{v_{1}, \ldots v_{N}\right\}$. We set

$$
\mathcal{P}=\{P: P \text { is an admissible polygon }\} .
$$

We also define the class

$$
\mathcal{R}=\left\{P \in \mathcal{P}: \partial P \text { is piecewise } C^{1}\right\},
$$

and we call regular admissible polygons the elements of $\mathcal{R}$. The difference between a general admissible polygon and a regular admissible polygon is that each vertex of the second is the endpoint of exactly two sides.

Given a polygon $P$ in $\mathbb{R}^{2}$, we define the set $V(P) \subseteq \mathbb{R}^{2}$ of the vertices of $P$ to be

$$
V(P)=\left\{x \in \partial P: \partial P \text { is not } C^{1} \text { at } x\right\} .
$$

We also define the functional $F_{\mathcal{R}}: \mathcal{P} \rightarrow \mathbb{R}$ in the following way:

$$
F_{\mathcal{R}}(P)= \begin{cases}\sum_{v \in V(P)} g\left(v^{-}(v), v^{+}(v)\right), & \text { if } P \in \mathcal{R} ; \\ +\infty, & \text { if } P \notin \mathcal{R} .\end{cases}
$$

Here, $v^{-}(v), v^{+}(v)$ denote the directions of the two sides intersecting in $v \in V(P)$. This functional will be identified with a functional $F_{\mathcal{R}}: L^{1}\left(\mathbb{R}^{2}\right) \rightarrow[0,+\infty]$ in the same spirit of (16).

We also set

$$
G=s c^{-}\left(F_{\mathcal{R}}\right),
$$

where $s c^{-}$denotes the sequential lower semi-continuous envelope, understood in the sense of the $L^{1}$-topology with uniform bounds on the perimeters, namely

$$
s c^{-}\left(F_{\mathcal{R}}\right)(E):=\inf \left\{\liminf _{n} F_{\mathcal{R}}\left(E_{n}\right): E_{n} \rightarrow E \text { in } L^{1}\left(\mathbb{R}^{2}\right), \sup _{n} \mathcal{H}^{1}\left(\partial E_{n}\right)<+\infty\right\} .
$$

REMARK 2.1 It can be easily checked that $G$ is finite only on (characteristic functions of) admissible polygons. Moreover, given an admissible polygon $P$, there always exists a sequence $\left(P_{n}\right)$ of regular polygons which converge to $P$ in $L^{1}\left(\mathbb{R}^{2}\right)$, and for which $\sup _{n} \mathcal{H}^{1}\left(\partial P_{n}\right)<+\infty$ and $\sup _{n} F_{\mathcal{R}}\left(P_{n}\right)<+\infty$. In fact, it is sufficient to take

$$
P_{n}=\left\{x \in P: \operatorname{dist}(x, \partial P) \leqslant \frac{1}{n}\right\} .
$$

Note that in general the sequence given by formula (18) does not recover the infimum in the definition of $G(E)$.

REMARK 2.2 Given an admissible polygon $P$, there always exists a sequence $\left(P_{n}\right)$ of regular polygons which converge to $P$ in $L^{1}\left(\mathbb{R}^{2}\right)$, and for which $G(P)=F_{\mathcal{R}}\left(P_{n}\right)$ for sufficiently large $n$. In fact, whenever the quantity $F_{\mathcal{R}}\left(P_{n}\right)$ remains bounded, it ranges over a finite set of numbers, and the infimum is always attained. 
Our main result is the following $\Gamma$-convergence theorem (for a general introduction to the subject we refer to $[11,13])$.

THEOREM 2.1 For $\varepsilon>0$, let $F_{\varepsilon}: L^{1}\left(\mathbb{R}^{2}\right) \rightarrow[0,+\infty]$ be the functional given by formula (16). Then

$$
\Gamma-\lim _{\varepsilon \rightarrow 0} F_{\varepsilon}=G
$$

with respect to the convergence in $L^{1}\left(\mathbb{R}^{2}\right)$ with uniform bound of the perimeter. By (19) we mean:

(i) (closure) if $\sup _{\varepsilon} \mathcal{H}^{1}\left(E_{\varepsilon}\right)<+\infty, \sup _{\varepsilon} F_{\varepsilon}\left(E_{\varepsilon}\right)<+\infty$ and $E_{\varepsilon} \rightarrow u$ in $L^{1}\left(\mathbb{R}^{2}\right)$ then there exists $P \in \mathcal{P}$ such that $u=\chi_{P}$;

(ii) ( $\Gamma$-lim inf inequality) for all $P \in \mathcal{P}$ and for all $E_{\varepsilon} \rightarrow P$ in $L^{1}\left(\mathbb{R}^{2}\right)$ with $\sup _{\varepsilon} \mathcal{H}^{1}\left(E_{\varepsilon}\right)<+\infty$, we have $G(P) \leqslant \liminf _{\varepsilon} F_{\varepsilon}\left(E_{\varepsilon}\right)$;

(iii) $\left(\Gamma\right.$-lim sup inequality) for all $P \in \mathcal{P}$ there exists $E_{\varepsilon} \rightarrow P$ in $L^{1}\left(\mathbb{R}^{2}\right)$ with $\sup _{\varepsilon} \mathcal{H}^{1}\left(E_{\varepsilon}\right)<+\infty$ such that $G(P)=\lim _{\varepsilon} F_{\varepsilon}\left(E_{\varepsilon}\right)$.

REMARK 2.3 (Convergence of minimum problems) From Theorem 2.1 we obtain the convergence of the minimum values of problems (13) and (14) to the minimum values

$$
\min \left\{G(P): P \in \mathcal{P},\left|E_{0} \Delta P\right| \leqslant \delta\right\}=\inf \left\{\sum_{v \in V(P)} g\left(v^{-}(v), v^{+}(v)\right): P \in \mathcal{R},\left|E_{0} \Delta P\right| \leqslant \delta\right\},
$$

and

$$
\min \left\{G(P)+\left|E_{0} \Delta P\right|: P \in \mathcal{P}\right\}=\inf \left\{\sum_{v \in V(P)} g\left(v^{-}(v), v^{+}(v)\right)+\left|E_{0} \Delta P\right|: P \in \mathcal{R}\right\},
$$

respectively, provided that there exists a sequence of minimizers with equi-bounded perimeter. This property is a well known result of $\Gamma$-convergence, once we notice that the equi-boundedness of the perimeters ensures compactness of the minimizing sequence (upon, possibly, a translation), and that the constraints or the additional terms are 'compatible' with $\Gamma$-convergence. To check this for problem (13), it is sufficient to notice that a slight modification of the argument in the proof of Theorem 2.1(iii) allows us to suppose $\left|E_{0} \Delta E\right| \leqslant \delta$. On the other hand, it is clear that the addition of the perturbation in (14) is compatible, since it is continuous with respect to the $L^{1}$-convergence.

REMARK 2.4 Theorem 2.1 remains valid if $F_{\varepsilon}$ takes the form

$$
F_{\varepsilon}(E)=\int_{\partial E}\left(\frac{1}{\varepsilon} \varphi(\nu)+\varepsilon \kappa^{2}\right) \mathrm{d} \mathcal{H}^{1}+c \mathcal{H}^{1}(\partial E),
$$

with $c>0$, i.e. if we add a term proportional to the length of $\partial E$. In this case, we similarly modify $F_{\mathcal{R}}(E)$ by setting

$$
F_{\mathcal{R}}(E)=\sum_{v \in V(E)} g\left(v^{-}(v), v^{+}(v)\right)+c \mathcal{H}^{1}(\partial E)
$$

on $\mathcal{R}$. Note that in this case the equi-boundedness condition on the perimeter is redundant.

We refer to Section 6 for the case when we drop the equi-boundedness condition on the perimeters and we consider the $L_{\text {loc }}^{1}$ convergence. We conclude this section by deducing a convergence result for the minimum problems in (1) as an example of application of Theorem 2.1. 
CoROllary 2.1 Let $\psi$ and $\bar{\psi}$ be as in the Introduction. Let $E_{0}$ be a bounded connected open set and let $E_{\varepsilon}$ be minimizers for the problems

$$
m_{\varepsilon}=\min \left\{\int_{\partial E}\left(\psi\left(v_{E}\right)+\varepsilon^{2} \kappa^{2}\right) \mathrm{d} \mathcal{H}^{1}: E_{0} \subseteq E\right\} .
$$

Then, to within a translation and a passage to a subsequence, $\left(E_{\varepsilon}\right)$ converges to a polygon $P$ which minimizes both

$$
m=\min \left\{\int_{\partial E} \bar{\psi}\left(v_{E}\right) \mathrm{d} \mathcal{H}^{1}: E_{0} \subseteq E\right\}
$$

and

$$
m^{(1)}=\min \left\{\sum_{v \in V(E)} g\left(v^{-}(v), v^{+}(v)\right): E_{0} \subseteq E, E \in \mathcal{R}\right\} .
$$

Proof. We just sketch the proof, including details only for the passages involving $\Gamma$-convergence.

By a relaxation argument (see [4]) and the density of sets with regular boundary we may suppose that $E_{\varepsilon}$ converges to a minimizer $\bar{E}$ of (21), which is connected since such is $E_{0}$. On the other hand, $E_{\varepsilon}$ is also a minimizer of

$$
m_{\varepsilon}^{(1)}=\min \left\{\int_{\partial E}\left(\frac{\psi\left(\nu_{E}\right)}{\varepsilon}+\varepsilon \kappa^{2}\right) \mathrm{d} \mathcal{H}^{1}-\frac{m}{\varepsilon}: E_{0} \subseteq E\right\} .
$$

Define $\varphi=\psi-\bar{\psi}$. By using the construction of Section 4 , it is easily seen that we have

$$
m \leqslant \int_{\partial E_{\varepsilon}} \bar{\psi}\left(v_{E_{\varepsilon}}\right) \mathrm{d} \mathcal{H}^{1} \leqslant m+o(\varepsilon),
$$

and that $E_{\varepsilon}$ is an $o(1)$-minimizer of

$$
\begin{aligned}
\tilde{m}_{\varepsilon}^{(1)} & =\min \left\{\int_{\partial E}\left(\frac{\psi\left(v_{E}\right)-\bar{\psi}\left(v_{E}\right)}{\varepsilon}+\varepsilon \kappa^{2}\right) \mathrm{d} \mathcal{H}^{1}: E_{0} \subseteq E\right\} \\
& =\min \left\{\int_{\partial E}\left(\frac{\varphi\left(v_{E}\right)}{\varepsilon}+\varepsilon \kappa^{2}\right) \mathrm{d} \mathcal{H}^{1}: E_{0} \subseteq E\right\} .
\end{aligned}
$$

We may apply Theorem 2.1 and Remark 2.4 as the perimeter of $E_{\varepsilon}$ is equi-bounded since $\psi \geqslant c$. We then obtain that $\bar{E}$ is a (convex) polygon minimizing both (21) and (22).

\subsection{Notation}

We introduce some preliminary notation and definitions.

Given a polygon $P$ in $\mathbb{R}^{2}$, we define a side of $P$ to be the closure of a component of $\partial P \backslash V(P)$; we also define

$$
\bar{s}(P)=\min \{\text { length of } s: s \text { is a side of } P\} .
$$

If $\gamma^{i}:\left[a_{i}, b_{i}\right] \rightarrow \mathbb{R}^{2} \quad i=1,2$ are two curves with $\gamma^{1}\left(b_{1}\right)=\gamma^{2}\left(a_{2}\right)$, we define $\gamma^{1} * \gamma^{2}$ : $\left[a_{1}, b_{1}+b_{2}-a_{2}\right] \rightarrow \mathbb{R}^{2}$ as

$$
\left(\gamma^{1} * \gamma^{2}\right)(t)= \begin{cases}\gamma^{1}(t) & t \in\left[a_{1}, b_{1}\right] \\ \gamma^{2}\left(t-b_{1}+a_{2}\right) & t \in\left[b_{1}, b_{1}+b_{2}-a_{2}\right] .\end{cases}
$$


Similarly, we define inductively

$$
\gamma^{1} * \cdots * \gamma^{k}=\left(\gamma^{1} * \cdots * \gamma^{k-1}\right) * \gamma^{k} .
$$

Given a curve $c:[a, b] \rightarrow \mathbb{R}^{2}$, we denote by $\operatorname{im}(c)$ its image. If $c$ is of class $C^{2}$, and $t \in[a, b]$ is such that $c^{\prime}(t) \neq 0$, we define $\kappa(c(t))$ to be the curvature of $c$ at $c(t)$.

Given two sequences $\left(A_{n}\right),\left(B_{n}\right)$ of subsets of $\mathbb{R}^{2}$ such that $A_{n} \cap B_{n}=\emptyset$ for all $n$, and given $v \in S^{1}$, we say that $\left(B_{n}\right)$ aligns with $\left(A_{n}\right)$ in the direction $v$ (or that $\left(B_{n}\right)$ and $\left(A_{n}\right)$ are in line with the direction $\nu$ ) if for every $\delta>0$ it is

$$
\left|\frac{x-y}{|x-y|}-v\right|<\delta, \quad \forall x \in A_{n}, \forall y \in B_{n}, \quad \text { for } n \text { sufficiently large. }
$$

We say that a family of curves $\gamma_{n}:\left(a_{n}, b_{n}\right) \rightarrow \mathbb{R}^{2}$ aligns in the direction $v$ if for every $\eta>0$ and for every sequence of pairs $\left(x_{n}, y_{n}\right), x_{n}, y_{n} \in \operatorname{im}\left(\gamma_{n}\right)$, with $\left|x_{n}-y_{n}\right|>\eta$, and such that $\gamma_{n}^{-1}\left(x_{n}\right)>\gamma_{n}^{-1}\left(y_{n}\right)$, the sequence $\left(x_{n}\right)$ aligns with $\left(y_{n}\right)$ in the direction $v$.

Given a piecewise $C^{1}$ curve $\gamma: S^{1} \rightarrow \mathbb{R}^{2}$, and given a point $x$ which does not belong to im $(\gamma)$, we define ind $(x, \gamma)$ to be the winding number of $\gamma$ around $x$, namely (in complex notation)

$$
\operatorname{ind}(\gamma, x)=\frac{1}{2 \pi \mathrm{i}} \int_{S^{1}} \frac{\dot{\gamma}(t)}{\gamma(t)-x} \mathrm{~d} t
$$

Finally, we say that two segments $\left[x_{1}, x_{2}\right],\left[y_{1}, y_{2}\right] \subseteq \mathbb{R}^{2}$ do not intersect transversally if

$$
\left(x_{1}, x_{2}\right) \cap\left(y_{1}, y_{2}\right)=\emptyset .
$$

Given $\theta_{1}, \theta_{2} \in S^{1}$, the sum $\theta_{1}+\theta_{2}$ will denote, unless it is explicitly remarked, the sum as elements of the group $S^{1}$ endowed with its natural structure (i.e the sum of $\mathbb{R}$ modulo $2 \pi$ ).

\section{The $\Gamma$-liminf inequality}

This section is devoted to the proof of the $\Gamma$ - lim inf inequality in Theorem 2.1.

We consider sequences $\left(E_{n}\right) \subseteq \mathbb{R}^{2}, \varepsilon_{n} \rightarrow 0^{+}$for which

$$
\begin{array}{ll}
\left(H_{1}\right) & \chi_{E_{n}} \rightarrow u \text { in } L^{1}\left(\mathbb{R}^{2}\right) ; \\
\left(H_{2}\right) & \sup _{n} \mathcal{H}^{1}\left(\partial E_{n}\right)<+\infty ; \\
\left(H_{3}\right) & \sup _{n} F_{\varepsilon_{n}}\left(E_{n}\right)<+\infty .
\end{array}
$$

Our first aim is to prove that the sequence $\left(E_{n}\right)$ converges in $L^{1}\left(\mathbb{R}^{2}\right)$ to some admissible polygon $P$. In fact we have the following result.

Proposition 3.1 Let $\varepsilon_{n} \rightarrow 0$ and let $\left(E_{n}\right)$ satisfy hypotheses $\left(H_{1}\right)-\left(H_{3}\right)$. Then there exists an admissible polygon $P \in \mathcal{P}$ such that $u=\chi_{P}$, and for which there holds

$$
G(u) \leqslant \liminf _{n} F_{\varepsilon_{n}}\left(E_{n}\right) .
$$

Before proving Proposition 3.1 we introduce some preliminary results. 
Lemma 3.1 Let $a, b, \delta \in \mathbb{R}, a<b, \delta>0$, and let $v_{i} \in \varphi^{-1}(0)$. Then for every curve $\eta:[a, b] \rightarrow$ $A_{\left(v_{i}, v_{i+1}\right)}$ of class $C^{1}$ with

$$
\eta(a)=v_{i}+\delta, \quad \eta(b)=v_{i+1}-\delta
$$

we have

$$
\int_{a}^{b}\left(\frac{1}{\varepsilon} \varphi(\eta(t))+\varepsilon\|\dot{\eta}(t)\|^{2}\right) \mathrm{d} t \geqslant g\left(v_{i}, v_{i+1}\right)+o_{\delta}(1),
$$

where $o_{\delta}(1) \rightarrow 0$ as $\delta \rightarrow 0$.

Proof. This is a simple consequence of the Young inequality: in fact we obtain

$$
\begin{aligned}
\int_{a}^{b}\left(\frac{1}{\varepsilon} \varphi(\eta(t))+\varepsilon\|\dot{\eta}(t)\|^{2}\right) \mathrm{d} t & \geqslant 2 \int_{a}^{b} \sqrt{\varphi(\eta(t))}|\dot{\eta}(t)| \mathrm{d} t \\
& \geqslant 2 \int_{v_{i}+\delta}^{v_{i+1}-\delta} \sqrt{\varphi(\eta(t))} \mathrm{d} t \geqslant 2 \int_{v_{i}}^{v_{i+1}} \sqrt{\varphi(\eta(t))} \mathrm{d} t+o_{\delta}(1),
\end{aligned}
$$

which is the desired inequality.

Now we consider a family of curves $\gamma_{n}: S^{1} \rightarrow \mathbb{R}^{2}$ of class $C^{2}$ with the following properties:

$$
\begin{gathered}
\sup _{n} \int_{S^{1}}\left(\frac{1}{\varepsilon_{n}} \varphi\left(\frac{\dot{\gamma}_{n}}{\left|\dot{\gamma}_{n}\right|}\right)+\varepsilon_{n}\left(\frac{\mathrm{d}}{\mathrm{d} t} \frac{\dot{\gamma}_{n}}{\left|\dot{\gamma}_{n}\right|}\right)^{2}\right) \mathrm{d} t=M<+\infty, \\
\sup _{n} \int_{S^{1}}\left|\dot{\gamma}_{n}\right| d t<+\infty .
\end{gathered}
$$

We suppose also that the curves $\gamma_{n}$ are parametrized proportionally to their arc length, namely that there holds

$$
\left|\dot{\gamma}_{n}(t)\right|=\frac{1}{2 \pi} \int_{S^{1}}\left|\dot{\gamma}_{n}\right| \mathrm{d} s ; \quad \text { for all } t \in S^{1} \text { and for all } n \in \mathbb{N} .
$$

We want to describe the limit shape of the curves $\gamma_{n}$ when $n \rightarrow+\infty$. In order to do this, we set for $\delta>0$

$$
S_{\delta}=S^{1} \backslash\left(\left[v_{1}-\delta, v_{1}+\delta\right] \cup \cdots \cup\left[v_{N}-\delta, v_{N}+\delta\right]\right),
$$

and

$$
C(\delta)=\inf _{\nu \in S_{\delta}} \varphi(v) .
$$

If $\eta:[a, b] \rightarrow S_{\delta}$ is a curve of class $C^{1}$, then there holds clearly

$$
\int_{a}^{b}\left(\frac{1}{\varepsilon} \varphi(\eta(t))+\varepsilon\|\dot{\eta}(t)\|^{2}\right) \mathrm{d} t \geqslant \frac{1}{\varepsilon}(b-a) C(\delta) ;
$$

hence, using (25) and (28) with $\eta=\dot{\gamma}_{n}$ and $\varepsilon=\varepsilon_{n}$, we deduce

$$
\mathcal{H}^{1}\left(\left\{t \in\left[0, T_{n}\right]: \dot{\gamma}_{n}(t) \in S_{\delta}\right\}\right) \leqslant \frac{\varepsilon_{n}}{C(\delta)} \int_{\dot{\gamma}_{n} \in S_{\delta}}\left(\frac{1}{\varepsilon_{n}} \varphi\left(\frac{\dot{\gamma}_{n}(t)}{\left|\dot{\gamma}_{n}\right|}\right)+\varepsilon_{n} \kappa^{2}\left(\gamma_{n}(t)\right)\right) \mathrm{d} t \leqslant \frac{\varepsilon_{n} M}{C(\delta)} .
$$


From this inequality we deduce the existence of a sequence $\delta_{n} \rightarrow 0$ such that

$$
\mathcal{L}^{1}\left(I_{n}\right) \rightarrow 0 \quad \text { as } n \rightarrow+\infty,
$$

where we have set

$$
I_{n}=\left\{t \in S^{1}: \frac{\dot{\gamma}_{n}(t)}{\left|\dot{\gamma}_{n}(t)\right|} \in S_{\delta_{n}}\right\} .
$$

Since $S_{\delta}$ is open, the components of $I_{n}$ are at most countable: denote by $I_{n}^{j}=\left(\sigma_{n}^{j}, \theta_{n}^{j}\right), j=$ $1, \ldots, k_{n}$, those components of $I_{n}$ for which $\dot{\gamma}_{n}\left(a_{n}^{j}\right) \neq \dot{\gamma}_{n}\left(b_{n}^{j}\right)$. From assumption $\left(H_{3}\right)$ and from Lemma 3.1 it follows that $\sup _{n} k_{n}<+\infty$ and so, passing to a subsequence, we can assume that $k_{n}=\bar{k}$ for all $n$. We also set

$$
J_{n}=S^{1} \backslash \bigcup_{j=1}^{\bar{k}} I_{n}^{j} .
$$

LEMMA 3.2 Let $\left(\theta_{n}^{h}, \sigma_{n}^{h+1}\right)$ be a component of $J_{n}$ such that

$$
\dot{\gamma}_{n}\left(\theta_{n}^{h}\right)=\left|\dot{\gamma}_{n}\right|\left(v_{i} \pm \delta_{n}\right), \quad \text { for some } v_{i} \in\left\{v_{1}, \ldots, v_{N}\right\} .
$$

Then $\left.\gamma_{n}\right|_{\left(\theta_{n}^{h}, \sigma_{n}^{h+1}\right)}$ aligns in the direction $v_{i}$.

Proof. Let $\eta>0$, and let $\alpha_{n}, \beta_{n} \in\left(\theta_{n}^{h}, \sigma_{n}^{h+1}\right)$ be such that $\left|\gamma_{n}\left(\alpha_{n}\right)-\gamma_{n}\left(\beta_{n}\right)\right|>\eta$. Then, since $\gamma_{n}$ is parametrized proportionally to the arc length, there holds

$$
\eta<\left|\gamma_{n}\left(\alpha_{n}\right)-\gamma_{n}\left(\beta_{n}\right)\right| \leqslant \int_{\alpha_{n}}^{\beta_{n}}\left|\dot{\gamma}_{n}(t)\right| \mathrm{d} t=\left|\dot{\gamma}_{n}\right| \mathcal{L}_{1}\left(\left(\alpha_{n}, \beta_{n}\right)\right),
$$

so in particular we have

$$
\frac{\eta}{\sup _{j}\left|\dot{\gamma}_{j}\right|} \leqslant \frac{\eta}{\left|\dot{\gamma}_{n}\right|}<\mathcal{L}_{1}\left(\left(\alpha_{n}, \beta_{n}\right)\right)<2 \pi .
$$

Hence by equation (26) the quantities $\mathcal{L}_{1}\left(\left(\alpha_{n}, \beta_{n}\right)\right)$ are uniformly bounded from above and from below. Set

$$
\rho_{n}=\int_{\left(\alpha_{n}, \beta_{n}\right) \backslash I_{n}} \dot{\gamma}_{n}(t) \mathrm{d} t ; \quad \tau_{n}=\int_{\left(\alpha_{n}, \beta_{n}\right) \cap I_{n}} \dot{\gamma}_{n}(t) \mathrm{d} t .
$$

Equations (26) and (29) imply that $\tau_{n} \rightarrow 0$ as $n \rightarrow+\infty$. We also have

$$
\int_{\left(\alpha_{n}, \beta_{n}\right) \backslash I_{n}} \dot{\gamma}_{n}(t) \mathrm{d} t=\left|\dot{\gamma}_{n}\right| \mathcal{L}_{1}\left(\left(\alpha_{n}, \beta_{n}\right) \backslash I_{n}\right) \nu_{i}+\int_{\left(\alpha_{n}, \beta_{n}\right) \backslash I_{n}}\left(\dot{\gamma}_{n}(t)-\left|\dot{\gamma}_{n}\right| v_{i}\right) \mathrm{d} t,
$$

so from (31) and the definition of $I_{n}$ we deduce

$$
\rho_{n}=\left|\dot{\gamma}_{n}\right| \mathcal{L}_{1}\left(\left(\alpha_{n}, \beta_{n}\right)\right) v_{i}+o(1) .
$$

From this expression and from the fact that $\rho_{n} \rightarrow 0$ it follows that

$$
\frac{\gamma_{n}\left(\beta_{n}\right)-\gamma_{n}\left(\alpha_{n}\right)}{\left|\gamma_{n}\left(\beta_{n}\right)-\gamma_{n}\left(\alpha_{n}\right)\right|}-v_{i}=\frac{\rho_{n}+\tau_{n}}{\left|\rho_{n}+\tau_{n}\right|}-v_{i}=\frac{\rho_{n}}{\left|\rho_{n}\right|}-v_{i}+o(1)=o(1) .
$$

This concludes the proof. 
The next lemma shows that $\gamma_{n}$, restricted to a component of $J_{n}$, converges uniformly to a segment in direction $v_{i}$ parametrized by arc length.

LEMMA 3.3 Let $\left(\theta_{n}^{h}, \sigma_{n}^{h+1}\right)$ be a component of $J_{n}$ as in Lemma 3.2. Then, given any $\rho>0$, there exists $n_{\rho} \in \mathbb{N}$ such that

$$
\left\|\gamma_{n}\left(\beta_{n}\right)-\gamma_{n}\left(\alpha_{n}\right)-\left|\dot{\gamma}_{n}\right|\left(\beta_{n}-\alpha_{n}\right) v_{i}\right\|<\rho, \quad \forall \alpha_{n}, \beta_{n} \in\left(\theta_{n}^{h}, \sigma_{n}^{h+1}\right), \quad \forall n \geqslant n_{\rho} .
$$

Proof. This follows easily from $\gamma_{n}\left(\beta_{n}\right)-\gamma_{n}\left(\alpha_{n}\right)=\rho_{n}+\tau_{n}$, equation (32), and the fact that $\tau_{n} \rightarrow 0$ as $n \rightarrow+\infty$.

Let us now introduce some additional notation. We define the class

$\mathcal{C}=\left\{\left\{\gamma^{1}, \ldots, \gamma^{k}\right\} \mid \gamma^{i}: S^{1} \rightarrow \mathbb{R}^{2}\right.$ is piecewise $C^{1}, \frac{\dot{\gamma}^{i}}{\left|\dot{\gamma}^{i}\right|} \in\left\{v_{1}, \ldots, v_{N}\right\}$ a.e. in $\left.S^{1}, i=1, \ldots, k\right\}$.

Let $\gamma=\left\{\gamma^{1}, \ldots, \gamma^{k}\right\} \in \mathcal{C}$. Then for all $i \operatorname{im}\left(\gamma^{i}\right)$ is composed by a finite number of segments with directions $v_{j_{1}}, \ldots, v_{j_{i}}$. We define $\tilde{F}: \mathcal{C} \rightarrow \mathbb{R}$ in the following way:

$$
\tilde{F}(\gamma)=\sum_{i=1}^{k} \sum_{h=1}^{j_{i}} g\left(v_{j_{h}}, v_{j_{h+1}}\right)
$$

Proposition 3.2 Let $\varepsilon_{n}$ and let $\left(E_{n}\right)$ satisfy hypotheses $\left(H_{1}\right)-\left(H_{3}\right)$ above. Let $\gamma_{n}^{j}, j=$ $1, \ldots, l$ (passing to a subsequence we can suppose that the number $l$ is independent of $n$ ) be parameterizations of the components of $\partial E_{n}$. Then there exist a polygon $P \in \mathcal{P}$ such that $u=\chi_{P}$, there exist integers $h, k, k \leqslant h \leqslant l$, and there exists $\gamma=\left\{\gamma^{1}, \ldots, \gamma^{k}\right\} \in \mathcal{C}$ with the following properties:

$\left(\Gamma_{1}\right) \gamma_{n}^{j} \rightarrow \gamma^{j}, j=1, \ldots, k$, uniformly on $S^{1}$, and $\gamma_{n}^{j} \rightarrow x^{j} \in \mathbb{R}^{2}, j=k+1, \ldots, h$, uniformly on $S^{1}$.

$\left(\Gamma_{2}\right)$ the segments of $\operatorname{im}(\gamma)$ do not intersect transversally;

$\left(\Gamma_{3}\right)$ for a.e. $x \in \mathbb{R}^{2}$, it is $\sum_{i=1}^{k} \operatorname{ind}\left(\gamma^{i}, x\right) \in\{0,1\}$, and $\chi_{P}(x)=\sum_{i=1}^{k} \operatorname{ind}\left(\gamma^{i}, x\right)$;

$\left(\Gamma_{4}\right) \tilde{F}(\gamma) \leqslant \liminf _{n} F_{\varepsilon_{n}}\left(E_{n}\right)$.

Proof. Let $i \in\{1, \ldots, h\}$, and consider the sequence of curves $\gamma_{n}^{i}$ which parameterize the $i$-th component of $\partial E_{n}$. This sequence satisfies conditions (25) and (26), hence we can repeat for them the constructions above. Let $J_{n}^{i}$ be the counterpart of the set $J_{n}$ for the curve $\gamma_{n}^{i}$. We can also suppose that the number of components of $J_{n}^{i}$ is a constant $k^{i}$ independent of $n$. From Lemma 3.3 it follows that

up to translation, $\gamma_{n}^{i} \rightarrow \gamma^{i}$ uniformly on $S^{1}, \quad$ for some curve $\gamma^{i} \in \mathcal{C}$,

or

up to translation, $\gamma_{n}^{i} \rightarrow x^{i}$ uniformly on $S^{1}, \quad$ for some point $x^{i} \in \mathbb{R}^{2}$. 
Up to a permutation of the indices, there exist $h, k \in \mathbb{N}, 0 \leqslant k \leqslant h \leqslant l$ such that $\left(\gamma_{n}^{1}\right), \ldots,\left(\gamma_{n}^{k}\right)$ converge uniformly in $S^{1}$ to some $\gamma^{1}, \ldots, \gamma^{k} \in \mathcal{C}$, and that $\left(\gamma_{n}^{k+1}\right), \ldots,\left(\gamma_{n}^{h}\right)$ converge uniformly in $S^{1}$ to some points $x^{k+1}, \ldots, x^{h} \in \mathbb{R}^{2}$. Define $\gamma$ to be $\gamma=\left\{\gamma^{1}, \ldots, \gamma^{k}\right\}$, so that also $\gamma \in \mathcal{C}$. Condition $\left(\Gamma_{1}\right)$ is automatically satisfied. Condition $\left(\Gamma_{2}\right)$ follows easily from the fact that the sets $E_{n}$ are of class $C^{2}$.

From equations (34) and (35) we deduce

$$
\mathcal{H}^{2}\left(B_{\gamma}\right)=0, \quad \text { where } \quad B_{\gamma}=\left(\bigcup_{i=1}^{k} \operatorname{im}\left(\gamma^{i}\right)\right) \cup\left(\bigcup_{i=k+1}^{h} x^{i}\right) .
$$

By the continuity of the winding number with respect to the uniform convergence we have

$$
\lim _{n} \sum_{i=1}^{h} \operatorname{ind}\left(\gamma_{n}^{i}, x\right)=\sum_{i=1}^{h} \operatorname{ind}\left(\gamma^{i}, x\right), \quad \text { for all } x \in \mathbb{R}^{2} \backslash B_{\gamma},
$$

hence, since the index is integer-valued there holds

$$
\sum_{i=1}^{h} \text { ind }\left(\gamma_{n}^{i}, x\right)=\sum_{i=1}^{k} \text { ind }\left(\gamma^{i}, x\right), \quad \text { for } n \text { large and for all } x \in \mathbb{R}^{2} \backslash B_{\gamma} .
$$

From this we can deduce that, setting

$$
P=\left\{x \in \mathbb{R}^{2} \backslash B_{\gamma}: \lim _{n} \sum_{i=1}^{h} \operatorname{ind}\left(\gamma_{n}^{i}, x\right)=1\right\},
$$

we have

This implies that

$$
\left\{\begin{array}{lll}
x \in P \quad & \Rightarrow \quad x \in E_{n} & \text { for } n \text { large } \\
x \notin P \quad \Rightarrow \quad x \notin E_{n} & \text { for } n \text { large. }
\end{array}\right.
$$

$$
\chi_{E_{n}} \rightarrow \chi_{P} \quad \text { as } n \rightarrow+\infty, \quad \text { a.e. in } \mathbb{R}^{2},
$$

and proves condition $\left(\Gamma_{3}\right)$. Property $\left(\Gamma_{4}\right)$ follows from Lemma 3.1.

Lemma 3.4 Suppose that $\gamma \in \mathcal{C}$ satisfies conditions $\left(\Gamma_{1}\right)$ and $\left(\Gamma_{2}\right)$ in Proposition 3.2. Then there exists a sequence of regular polygons $\left(P_{n}\right) \subseteq \mathcal{R}$ such that

$$
\chi_{P_{n}} \rightarrow \chi_{P} \quad \text { in } L^{1}\left(\mathbb{R}^{2}\right) ; \quad F_{\mathcal{R}}\left(P_{n}\right) \leqslant \tilde{F}(\gamma) .
$$

Proof. For the proof of this Lemma we refer to [14].

Finally, we are in position to prove Proposition 3.1.

Proof of Proposition 3.1. Let $P$ be the polygon given by Proposition 3.2, and let $\left(P_{n}\right) \subseteq \mathcal{R}$ be the sequence of regular polygons given by Lemma 3.4. Then, by equation (37) and by property $\left(\Gamma_{4}\right)$ there holds

$$
F_{\mathcal{R}}\left(P_{n}\right) \leqslant \tilde{F}(\gamma) \leqslant \liminf _{n} F_{\varepsilon_{n}}\left(E_{n}\right) .
$$

Finally, by the definition of $G$ we have

$$
G(P) \leqslant \liminf _{n} F_{\mathcal{R}}\left(P_{n}\right) \leqslant \liminf _{n} F_{\varepsilon_{n}}\left(E_{n}\right) .
$$

This concludes the proof. 


\section{The $\Gamma$-limsup inequality}

The goal of this section is to prove the $\Gamma$-lim sup inequality in Theorem 2.1. Starting with a regular admissible polygon $P$, we modify it near its vertices and we obtain a sequence of sets $E_{n}$ of class $C^{2}$ which converge to $P$ and such that $F_{\varepsilon_{n}}\left(E_{n}\right)$ is as small as possible. Then we treat the general case of an admissible polygon by approximating it with regular polygons.

Proposition 4.1 Let $P \in \mathcal{R}$ be an admissible regular polygon. Then, given any sequence $\varepsilon_{n} \rightarrow$ $0^{+}$, there exists a sequence of sets $\left(E_{n}\right)$ of class $C^{2}$ such that

$$
\chi_{E_{n}} \rightarrow \chi_{P} \text { in } L^{1}\left(\mathbb{R}^{2}\right) ; \quad \limsup _{n} F_{\varepsilon_{n}}\left(E_{n}\right) \leqslant F_{\mathcal{R}}(P) .
$$

Proof. Let $v$ be a vertex of $P$ : since $P$ is regular, there are exactly two sides of $P$ intersecting $v$. Without loss of generality, we can suppose that the directions of these sides, which we denote by $l_{1}$ and $l_{2}$, are $v_{1}$ and $\nu_{2}$ respectively. Let $\lambda:\left(-\frac{1}{2}\left|l_{1}\right|, \frac{1}{2}\left|l_{2}\right|\right) \rightarrow \mathbb{R}^{2}$ be defined by

$$
\lambda(t)= \begin{cases}v-t v_{1}, & t \in\left[-\frac{1}{2} l_{1}, 0\right] ; \\ v+t v_{2}, & t \in\left[0, \frac{1}{2} l_{2}\right] .\end{cases}
$$

The curve $\lambda$ defined in this way parametrizes part of $l_{1}$ for $t<0$ and part of $l_{2}$ for $t>0$. Our aim is to find a sequence of regular curves $\lambda_{n}:\left[-\frac{1}{2}\left|l_{1}\right|, \frac{1}{2}\left|l_{2}\right|\right] \rightarrow \mathbb{R}^{2}$ with the following properties:

$$
\begin{gathered}
\lambda_{n} \rightarrow \lambda \quad \text { uniformly on }\left[-\frac{1}{2}\left|l_{1}\right|, \frac{1}{2}\left|l_{2}\right|\right] ; \\
\lim _{n} \int_{\left(-\frac{1}{2}\left|l_{1}\right|, \frac{1}{2}\left|l_{2}\right|\right)}\left(\frac{1}{\varepsilon_{n}} \varphi\left(\frac{\dot{\lambda}_{n}}{\left|\dot{\lambda}_{n}\right|}\right)+\varepsilon_{n} \kappa^{2}\left(\lambda_{n}\right)\right) \mathrm{d} t=g\left(v_{1}, v_{2}\right) .
\end{gathered}
$$

Since $\varphi$ is assumed to be of class $C^{1}$ in $S^{1} \backslash\left\{v_{1}, \ldots, v_{N}\right\}$, the following Cauchy problem:

$$
\left\{\begin{array}{l}
y^{\prime}(t)=\sqrt{\varphi(y(t))}\left(\begin{array}{rr}
0 & -1 \\
1 & 0
\end{array}\right) y(t) \\
y(0)=\frac{v_{1}+v_{2}}{2}
\end{array}\right.
$$

admits a unique maximal solution $u:(a, b) \rightarrow S^{1}$, with $-\infty \leqslant a<0,0<b \leqslant+\infty$. It is immediate to check that $u$ is a $C^{1}$ increasing function which tends to $v_{1}$ (respectively, $v_{2}$ ) as $t \rightarrow a$ (respectively, $t \rightarrow b$ ).

For every $c, d \in(a, b)$, with $c<0<d$ ( $c$ and $d$ will be taken sufficiently close to $a$ and $b$ ), define $e=c-\left(u(c)-v_{1}\right)$ and $f=d+\left(v_{2}-u(d)\right)$; note that $e<c<d<f$. We can find a nondecreasing function $\eta:[e, f] \rightarrow A_{\left(v_{1}, v_{2}\right)}$ of class $C^{1}$, such that

$$
\begin{array}{cl}
\eta(e)=v_{1} ; & \dot{\eta}(e)=0 ; \\
\eta(f)=v_{1} ; & \dot{\eta}(f)=0 ;
\end{array}
$$




$$
\begin{cases}\eta(t)=u(t), & t \in(c, d) ; \\ |\dot{\eta}(t)|<2\left|u(c)-v_{1}\right|, & t \in(e, c) ; \\ |\dot{\eta}(t)|<2\left|v_{2}-u(d)\right|, & t \in(d, f) .\end{cases}
$$

For $\varepsilon>0$, let $\eta_{\varepsilon}$ denote the unique continuous extension of $\eta$ to the interval $\left[-\frac{1}{\varepsilon} \frac{1}{2}\left|l_{1}\right|, \frac{1}{\varepsilon} \frac{1}{2}\left|l_{2}\right|\right]$ for which

$$
\eta_{\varepsilon}(t)= \begin{cases}v_{1}, & t \in\left[-\frac{1}{\varepsilon} \frac{1}{2}\left|l_{1}\right|, e\right] ; \\ v_{2}, & t \in\left[f, \frac{1}{\varepsilon} \frac{1}{2}\left|l_{2}\right|\right] .\end{cases}
$$

Finally, for $\varepsilon_{n} \rightarrow 0^{+}$, define $\lambda_{n}:\left[-\frac{1}{2}\left|l_{1}\right|, \frac{1}{2}\left|l_{2}\right|\right] \rightarrow \mathbb{R}^{2}$ to be

$$
\lambda_{n}(t)=v+\int_{0}^{t} \eta_{\varepsilon_{n}}\left(\frac{s}{\varepsilon_{n}}\right) \mathrm{d} s, \quad t \in\left[-\frac{1}{2}\left|l_{1}\right|, \frac{1}{2}\left|l_{2}\right|\right] .
$$

Since $\eta_{\varepsilon_{n}}$ is an $S^{1}$-valued curve of class $C^{1}$, it follows that $\lambda_{n}$ is of class $C^{2}$ and is parametrized by arc length. For $t<0$ it turns out that

$$
\begin{aligned}
\lambda_{n}(t)-\lambda(t) & =v+\int_{0}^{t} \eta_{\varepsilon_{n}}\left(\frac{s}{\varepsilon_{n}}\right) \mathrm{d} s-v-t v_{1} \\
& =\int_{0}^{\varepsilon_{n} e} \eta_{\varepsilon_{n}}\left(\frac{s}{\varepsilon_{n}}\right) \mathrm{d} s+\int_{\varepsilon_{n} e}^{t} \eta_{\varepsilon_{n}}\left(\frac{s}{\varepsilon_{n}}\right) \mathrm{d} s-t v_{1} .
\end{aligned}
$$

Since $\left|\eta_{\varepsilon_{n}}\right|=1$, and since $\eta_{\varepsilon_{n}}(t)=v_{1}$ for $t<e$, it follows that

$$
\lambda_{n}(t)-\lambda(t) \rightarrow 0, \quad \text { uniformly for } t \in\left[-\frac{1}{2}\left|l_{1}\right|, 0\right] .
$$

In the same way one can show that

$$
\lambda_{n}(t)-\lambda(t) \rightarrow 0, \quad \text { uniformly for } t \in\left[0, \frac{1}{2}\left|l_{1}\right|,\right],
$$

so we have proved (39).

Using the definition of $\lambda_{n}$ and the change of variable $\frac{s}{\varepsilon_{n}}=y$, we find

$$
\int_{-\frac{1}{2}\left|l_{1}\right|}^{\frac{1}{2}\left|l_{2}\right|}\left(\frac{1}{\varepsilon_{n}} \varphi\left(\frac{\dot{\lambda}_{n}}{\left|\dot{\lambda}_{n}\right|}\right)+\varepsilon_{n} \kappa^{2}\left(\lambda_{n}\right)\right) \mathrm{d} s=\int_{-\frac{1}{2} \frac{1}{\varepsilon_{n}}\left|l_{1}\right|}^{\frac{1}{2} \frac{1}{\varepsilon_{n}}\left|l_{2}\right|}\left(\varphi\left(\eta_{\varepsilon_{n}}\right)+\left(\dot{\eta}_{\varepsilon_{n}}\right)^{2}\right) \mathrm{d} y
$$

then, taking into account equation (45), one has

$$
\int_{-\frac{1}{2} \frac{1}{\varepsilon_{n}}\left|l_{1}\right|}^{\frac{1}{2} \frac{1}{\varepsilon_{n}}\left|l_{2}\right|}\left(\varphi\left(\eta_{\varepsilon_{n}}\right)+\left(\dot{\eta}_{\varepsilon_{n}}\right)^{2}\right) \mathrm{d} t=\int_{e}^{f}\left(\varphi\left(\eta_{\varepsilon_{n}}\right)+\left(\dot{\eta}_{\varepsilon_{n}}\right)^{2}\right) \mathrm{d} t .
$$

Dividing the interval $(e, f)$ into $(e, c),(c, d)$ and $(d, f)$, by equation (41) we get

$$
\begin{aligned}
\int_{e}^{f}\left(\varphi\left(\eta_{\varepsilon_{n}}\right)+\left(\dot{\eta}_{\varepsilon_{n}}\right)^{2}\right) \mathrm{d} t \leqslant & |c-e|\left(\sup _{(e, c)} \varphi+\sup _{(e, c)} \dot{\eta}_{\varepsilon_{n}}^{2}\right) \\
& +g\left(\nu_{1}, \nu_{2}\right)+|f-d|\left(\sup _{(d, f)} \varphi+\sup _{(d, f)} \dot{\eta}_{\varepsilon_{n}}^{2}\right) .
\end{aligned}
$$


Using the expression of $e, f$, and taking into account (44), we deduce

$$
\begin{aligned}
\int_{e}^{f}\left(\varphi\left(\eta_{\varepsilon_{n}}\right)+\left(\dot{\eta}_{\varepsilon_{n}}\right)^{2}\right) \mathrm{d} t \leqslant & g\left(\nu_{1}, \nu_{2}\right)+\left|u(c)-v_{1}\right|\left(\sup _{(e, c)} \varphi+4\left|u(c)-v_{1}\right|^{2}\right) \\
& +\left|\nu_{2}-u(d)\right|\left(\sup _{(e, c)} \varphi+4\left|\nu_{2}-u(d)\right|^{2}\right) .
\end{aligned}
$$

Hence, choosing $c=c(n)$ and $d=d(n)$ depending on $n$ and such that

$$
\left|u(c)-v_{1}\right|+\left|v_{2}-u(d)\right| \rightarrow 0 \quad \text { as } n \rightarrow+\infty,
$$

also (40) follows.

Now consider a component $\Theta$ of $\partial P$. Let $v_{1}, \ldots, v_{i_{\Theta}}$ denote an ordering of the vertices of $\Theta$ along the parametrization of $\lambda$, and let $\lambda_{j}$ be the curve defined above corresponding to the vertex $v_{j}, j=1, \ldots, i_{\Theta}$. Then we can choose as parametrization for $\Theta$ the piecewise- $C^{2}$ curve $\lambda_{\Theta}$ given by

$$
\lambda_{\Theta}=\lambda_{1} * \cdots * \lambda_{i_{\Theta}} .
$$

For $j \in\left\{1, \ldots, i_{\Theta}\right\}$, let $\lambda_{j, n}$ be a sequence of curves which satisfy (39) and (40) with $\lambda=\lambda_{j}$ and $v^{-}\left(v_{j}\right), v^{+}\left(v_{j}\right)$ instead of $v_{1}$ and $v_{2}$. If we consider the sequence of curves

$$
\lambda_{p, n}=\lambda_{1, n} * \cdots * \lambda_{i_{\Theta}, n}, \quad n \in \mathbb{N},
$$

they will converge uniformly to $\lambda_{\Theta}$ on their domain $\left(a_{\Theta}, b_{\Theta}\right)$. In general the curve $\lambda_{\Theta, n}$ is not closed, but since $\lambda_{\Theta}$ is closed there holds

$$
\lambda_{\Theta, n}\left(a_{\Theta}\right)-\lambda_{\Theta, n}\left(b_{\Theta}\right) \rightarrow 0, \quad \text { as } n \rightarrow+\infty .
$$

Consider the curve $\lambda_{1, n}$. Since the directions of its two rectilinear parts are linearly independent, it is sufficient to modify slightly the length of these parts in such a way that $\lambda_{\Theta, n}$ transforms into a closed curve $\bar{\lambda}_{\Theta, n}$.

Repeating this procedure for all the components of $\partial P$ we obtain a set $E_{n}$ whose boundary is parametrized by the union of the curves $\left(\bar{\lambda}_{\Theta, n}\right)_{\Theta}$. The sequence $E_{n}$ will satisfy the required properties in the proposition.

REMARK 4.1 From the proof of Proposition 4.1 it follows that we can choose $\lambda_{n}$ satisfying (40) and

$\lambda_{n}$ coincides with $\lambda$ in a neighbourhood of $\left\{-\frac{1}{2}\left|l_{1}\right|, \frac{1}{2}\left|l_{2}\right|\right\} ; \quad\left\|\lambda_{n}-\lambda\right\|_{\infty} \leqslant 2(|e(n)|+|f(n)|) \varepsilon_{n}$,

where $e(n)=c(n)-u(c(n))+v_{1}$ and $f(n)=d(n)+u(c(n))-v_{2}$.

As an immediate consequence of Proposition 4.1 we have the following corollary.

COROLLARY 4.1 ( $\Gamma$-lim sup inequality) Let $P \in \mathcal{P}$ be an admissible polygon. Then, for every $\left(\varepsilon_{n}\right)$ with $\varepsilon_{n} \rightarrow 0^{+}$there exists a sequence of sets $E_{n}$ of class $C^{2}$ such that

$$
E_{n} \rightarrow P \text { in } L^{1}\left(\mathbb{R}^{2}\right) \quad \text { and } \quad \lim \sup _{n} F_{\varepsilon_{n}}\left(E_{n}\right) \leqslant G(P) .
$$


Proof. By Remark 2.2, there exists a sequence $\left(P_{k}\right)_{k} \subseteq \mathcal{R}$ of regular polygons such that

$$
\chi_{P_{k}} \rightarrow \chi_{P} \text { in } L^{1}\left(\mathbb{R}^{2}\right) ; \quad \limsup _{k} F_{\mathcal{R}}\left(P_{k}\right)=G(P) ; \quad \sup _{k} \mathcal{H}^{1}\left(\partial P_{k}\right)<+\infty .
$$

Then, by Proposition 4.1, for every $k \in \mathbb{N}$ there exists a sequence $\left(E_{n}^{k}\right)$ of sets of class $C^{2}$ such that

$$
E_{n}^{k} \rightarrow P_{k} ; \quad F_{\varepsilon_{n}}\left(E_{n}^{k}\right) \rightarrow F_{\mathcal{R}}\left(P_{k}\right), \quad \text { as } n \rightarrow+\infty .
$$

Hence we can choose a sequence of natural numbers $n(k)$ with $n\left(k_{2}\right)>n\left(k_{1}\right)$ if $k_{2}>k_{1}$ such that

$$
\left\|\chi_{E_{n}^{k}}-\chi_{P_{k}}\right\|_{L^{1}\left(\mathbb{R}^{2}\right)} \leqslant \frac{1}{k}, \quad F_{\varepsilon_{n}}\left(E_{n}^{k}\right) \leqslant F_{\mathcal{R}}\left(P_{k}\right)+\frac{1}{k} .
$$

So, if we choose

$$
E_{n}=E_{n}^{k}, \quad \text { for } n(k) \leqslant n<n(k+1),
$$

the sequence $\left(E_{n}\right)$ satisfies the desired properties.

\section{Some local cases}

In this section we study some specific cases for which the $\Gamma$-limit $G$ has a local expression, namely it is the sum over the vertices of an energy depending only on each single vertex.

\subsection{A non-symmetric case}

In this section we treat the following particular case. We assume that the function $\varphi$ satisfies the conditions

(i) $\varphi \in C^{1}\left(S^{1}\right)$;

(ii) $\varphi^{-1}(0)=\left\{v_{1}, \ldots, v_{N}\right\}$, and for all $i$ we have $-v_{i} \notin \varphi^{-1}(0)$ if $v_{i} \in \varphi^{-1}(0)$.

Under these hypotheses we will prove that $\Gamma-\lim _{\varepsilon \rightarrow 0} F_{\varepsilon}$ has a local expression. Namely, to every vertex of an admissible polygon $P$ is associated a quantity $E(v)$, and $\Gamma-\lim _{\varepsilon \rightarrow 0} F_{\varepsilon}(P)$ is the sum of $E(v)$ over the vertices $v$ of $P$, see Proposition 5.4. In order to state this result precisely we introduce some additional notation.

Let $P$ be an admissible polygon, and let $v$ be a vertex of $P$. Let $l_{1}, \ldots, l_{2 k}$ be the sides of $P$ which intersect at $v$. If condition (i) above is satisfied, then for each of these segments $l_{j}, j=$ $1, \ldots, 2 k$, is uniquely determined a tangent direction $v\left(l_{j}\right)=v_{i_{j}} \in \varphi^{-1}(0)$.

To each $l_{j}$ we can associate an orientation $\sigma_{v}\left(l_{j}\right)$ with respect to $v$, namely we set

$$
\left\{\begin{array}{ll}
\sigma_{v}\left(l_{j}\right)=-1, & \text { if } l_{j} \text { is oriented toward } v ; \\
\sigma_{v}\left(l_{j}\right)=1, & \text { if }-l_{j} \text { is oriented toward } v,
\end{array} \quad j=1, \ldots, 2 k .\right.
$$

If the segments $l_{1}, \ldots, l_{2 k}$, are ordered in such a way that $\sigma_{v}\left(l_{j}\right) v_{i_{1}}<\sigma_{v}\left(l_{j}\right) v_{i_{2}}<\cdots<\sigma_{v}\left(l_{j}\right) v_{i_{2 k}}$, then clearly it must be

$$
\sigma_{v}\left(l_{j}\right) \cdot \sigma_{v}\left(l_{j+1}\right)=-1, \quad j=1, \ldots, 2 k-1, \quad \text { and } \quad \sigma_{v}\left(l_{2 k}\right) \cdot \sigma_{v}\left(l_{1}\right)=-1 .
$$


Definition 5.1 An admissible decomposition $\omega$ of $v$ is a partition of $l_{1}, \ldots, l_{2 k}$ in pairs $\left(l_{i}^{-}, l_{i}^{+}\right)$, $i=1, \ldots, k$, such that

$$
\sigma_{v}\left(l_{i}^{-}\right)=-1, \quad \sigma_{v}\left(l_{i}^{+}\right)=1 ; \quad i=1, \ldots, k,
$$

and

$$
\left[v\left(l_{i}^{-}\right), v\left(l_{i}^{+}\right)\right] \cap\left[v\left(l_{j}^{-}\right), v\left(l_{j}^{+}\right)\right], \quad i, j=1, \ldots, k, \quad i \neq j,
$$

where $\left[v\left(l_{h}^{-}\right), v\left(l_{h}^{+}\right)\right]$in the above formula denotes the segment in $\mathbb{R}^{2}$ joining $v\left(l_{h}^{-}\right)$and $v\left(l_{h}^{+}\right)$. We set also

$$
\Omega_{v}=\{\omega \mid \omega \text { is an admissible decomposition for } v\} .
$$

REMARK 5.2 Every vertex $v \in V(P)$ admits an admissible decomposition. In fact, if the vectors $v_{i_{1}}, \ldots, v_{i_{2 k}}$, are ordered in such a way that $v_{i_{1}}<v_{i_{2}}<\cdots<v_{i_{2 k}}$, then one can take

$$
l_{i}^{-}=l_{2 i-1}, \quad l_{i}^{+}=l_{2 i} \quad i=1, \ldots, k .
$$

To each admissible decomposition $\omega=\left\{\left(l_{i}^{-}, l_{i}^{+}\right)\right\}_{i}$ of a vertex $v$, we associate the energy $\psi(\omega)$ defined by

$$
\psi(\omega)=\sum_{i=1}^{k} g\left(v\left(l_{i}^{-}\right), v\left(l_{i}^{+}\right)\right),
$$

and we define

$$
E(v)=\min \left\{\psi(\omega) \mid \omega \in \Omega_{v}\right\} .
$$

Lemma 5.3 Let $\gamma \in \mathcal{C}$ satisfy conditions $\left(\Gamma_{2}\right)$ and $\left(\Gamma_{3}\right)$ in Proposition 3.2, and let $P$ be the polygon associated to $\gamma$ from $\left(\Gamma_{3}\right)$. Let $v \in V(P)$ and let $l_{1}, \ldots, l_{2 k}$ be the segments of $\gamma$ which intersect $v$. Let $l_{1}^{-}, \ldots, l_{k}^{-}$be the segments of $\left\{l_{1}, \ldots, l_{2 k}\right\}$ which are oriented toward $v$, and let $l_{1}^{+}, \ldots, l_{k}^{+}$ be the elements of $\left\{l_{1}, \ldots, l_{2 k}\right\}$ which, following the parametrization of $\gamma$, are after $l_{1}^{-}, \ldots, l_{k}^{-}$ respectively. Then $\omega_{v}^{\gamma}=\left(l_{j}^{-}, l_{j}^{+}\right), j=1, \ldots, k$, is an admissible decomposition of $v$.

Proof. Property $\left(A D_{1}\right)$ is immediate to verify. Condition $\left(A D_{2}\right)$ is equivalent to the fact that adjacent sides must have opposite orientations.

Proposition 5.4 Suppose $\varphi$ satisfies conditions (i) and (ii) above and let $P$ be an admissible polygon. Then

$$
G\left(\chi_{P}\right)=\sum_{v \in V(P)} E(v) .
$$

Proof. Let us prove first the $\Gamma$-liminf inequality. Let $\varepsilon_{n} \rightarrow 0$, let $\left(E_{n}\right)$ satisfy hypotheses $\left(H_{1}\right)-$ $-\left(H_{3}\right)$, and let $u=\chi_{P}$. Let $\gamma \in \mathcal{C}$ be given by Proposition 3.2. Then, if $\omega_{v}^{\gamma}$ is given by Lemma 5.3, there holds

$$
\tilde{F}(\gamma)=\sum_{v \in V(P)} E\left(\omega_{v}^{\gamma}\right) .
$$


Finally, using equation (48) and property $\left(\Gamma_{4}\right)$ in Proposition 3.2 we get

$$
\sum_{v \in V(P)} E(v) \leqslant \sum_{v \in V(P)} E\left(\omega_{\gamma}^{v}\right)=\tilde{F}(\gamma) \leqslant \liminf _{n} F_{\varepsilon_{n}}\left(E_{n}\right) .
$$

This proves the $\Gamma$-lim inf inequality; let us now turn to the $\Gamma$-lim sup inequality.

Let $v \in V(P)$ and let $\bar{\omega}_{v}$ be an admissible decomposition of $v$ which realizes the minimum energy, namely for which

$$
\psi\left(\bar{\omega}_{v}\right)=E(v) .
$$

The set of the admissible decompositions $\bar{\omega}_{v}$, when $v$ ranges over $V(P)$, determines an element $\gamma \in \mathcal{C}$ in the following way.

Given a side $l^{1}$ of $P$, are uniquely determined two vertices $v_{1}$ and $v_{2}$ and two indices $i_{1}$ and $i_{2}$ for which, if we set $\bar{\omega}_{1}=\left\{\left(l_{i, 1}^{+}, l_{i, 1}^{-}\right)\right\}_{i}$ and $\bar{\omega}_{2}=\left\{\left(l_{i, 2}^{+}, l_{i, 2}^{-}\right)\right\}_{i}$, we have

$$
l^{1}=l_{i_{1}, 1}^{+}=l_{i_{2}, 2}^{-} .
$$

Let $l^{2}=l_{i_{2}, 2}^{-}$; reasoning as above, there exist an unique vertex $v_{3}$ an unique index $i_{3}$ for which, if we set $\bar{\omega}_{3}=\left\{\left(l_{i, 3}^{+}, l_{i, 3}^{-}\right)\right\}_{i}$, there holds

$$
l^{2}=l_{i_{2}, 2}^{+}=l_{i_{3}, 3}^{-} .
$$

Continuing in this way, we obtain a first segment $l^{j_{1}}$ for which $l^{j_{1}}=l_{i_{1}, 1}^{-}$. Let $c^{i}:\left[\alpha^{i}, \beta^{i}\right] \rightarrow \mathbb{R}^{2}$, $i=1, \ldots, j$ be parameterizations of the sides $l^{i}$, and consider the closed curve $\gamma^{1}$ defined by

$$
\gamma^{1}=c^{1} * \cdots * c^{j} .
$$

Up to reparameterizations, we can suppose that $\gamma^{1}$ is defined on $S^{1}$. In the same way, we define the curves $\gamma^{2}, \ldots, \gamma^{k}: S^{1} \rightarrow \mathbb{R}^{2}$ until all the remaining sides of $P$ are considered.

Now we fix a number $M>0$, a sequence of positive numbers $\delta_{n}$ converging to zero, and we consider the set

$$
A_{n}=\left\{\cup \bar{B}_{M \delta_{n}}(v) \mid v \in V(P)\right\} .
$$

Let $\gamma^{1}$ be the curve defined above, and let $\xi_{n}^{1}=\left\{t \in S^{1}: \gamma^{1}(t) \in A_{n}\right\}$. The set $\xi_{n}^{1}$ is a finite union of closed intervals $\left[\alpha_{n}^{1, i}, \beta_{n}^{1, i}\right], i=1, \ldots, j_{1}$, and we denote by $\left(\sigma_{n}^{1, i}, \tau_{n}^{1, i}\right), i=1, \ldots, j_{1}$, the components of $S^{1} \backslash \xi_{n}^{1}$, where we have taken $\sigma_{n}^{1, i}=\beta_{n}^{1, i}$. Setting $\bar{c}_{n}^{1, i}=\left.\gamma^{1}\right|_{\left[\alpha_{n}^{1, i}, \beta_{n}^{1, i}\right]}$, and $\hat{c}_{n}^{1, i}=\left.\gamma^{1}\right|_{\left[\sigma_{n}^{1, i}, \tau_{n}^{1, i}\right]}$, it is clear that

$$
\gamma^{1}=\bar{c}_{n}^{1,1} * \hat{c}_{n}^{1,1} * \bar{c}_{n}^{1,2} * \cdots * \bar{c}_{n}^{1, j_{1}} * \hat{c}_{n}^{1, j_{1}} .
$$

Of course, we can write a similar expression for $\gamma^{2}, \ldots, \gamma^{k}$.

We observe that the maps $\bar{c}_{n}^{i, l}, i=1, \ldots, k, l=1, \ldots, j_{i}$, are union of two rectilinear curves with directions $v_{-}^{i, l}$ and $v_{+}^{i, l}$ (following the order of the parametrization), while the curves $\hat{c}_{n}^{i, l}$ are rectilinear with direction $v_{+}^{i, l}$.

We define also the curves

$$
\tilde{c}_{n}^{i, l}(t)=\bar{c}_{n}^{i, l}(t)+\delta_{n}\left(v_{-}^{i, l}+v_{+}^{i, l}\right), \quad t \in\left[\alpha_{n}^{i, l}, \beta_{n}^{i, l}\right] ;
$$


where the above sum $v_{-}^{i, l}+v_{+}^{i, l}$ is now a sum of elements in $\mathbb{R}^{2}$. It follows from property $\left(A D_{2}\right)$ that the images of the curves $\tilde{c}_{n}^{i, l}$ are all disjoint when $i$ varies from 1 to $k$, and $l$ varies from 1 to $j_{i}$. We have also

$$
\frac{\left(\tilde{c}_{n}^{i, l}\right)^{\prime}\left(\beta_{n}^{i, l}\right)}{\left|\left(\tilde{c}_{n}^{i, l}\right)^{\prime}\left(\beta_{n}^{i, l}\right)\right|}=v_{+}^{i, l}=v_{-}^{i, l+1}=\frac{\left(\tilde{c}_{n}^{i, l+1}\right)^{\prime}\left(\alpha_{n}^{i, l+1}\right)}{\left|\left(\tilde{c}_{n}^{i, l+1}\right)^{\prime}\left(\alpha_{n}^{i, l+1}\right)\right|}, \quad \text { for all } i=1, \ldots, k, l=1, \ldots, j_{i} .
$$

Now we choose a function $\eta:[0,1] \rightarrow[0,1]$ of class $C^{\infty}$ and which satisfies the following properties:

$$
\left\{\begin{array}{l}
\eta=0 \text { in a neighbourhood of } 0 \\
\eta=1 \text { in a neighbourhood of } 1 \\
\eta^{\prime} \geqslant 0 ; \quad\left|\eta^{\prime}\right| \leqslant 2 ; \quad\left|\eta^{\prime \prime}\right| \leqslant 4
\end{array}\right.
$$

and for $a, b>0$, let $\eta_{a, b}:[0,1] \rightarrow \mathbb{R}^{2}$ be defined by

$$
\eta_{a, b}(t)=\left(\begin{array}{c}
a t \\
b \eta(t)
\end{array}\right) ; \quad t \in[0,1] .
$$

Using simple computations, one can check that

$$
\left|\kappa\left(\eta_{a, b}(t)\right)\right| \leqslant 4 \frac{b}{a}, \quad \text { for all } t \in[0,1] .
$$

We recall $\kappa\left(\eta_{a, b}(t)\right)$ denotes the curvature of $\eta_{a, b}$ at $\eta_{a, b}(t)$.

Fix $i \in\{1, \ldots, k\}, l \in\left\{1, \ldots, j_{i}\right\}$, and consider the points $\tilde{c}_{n}^{i, l}\left(\beta_{n}^{i, l}\right)$ and $\tilde{c}_{n}^{i, l+1}\left(\alpha_{n}^{i, l+1}\right)$; then by equation (50) there exist unique numbers $a, b>0$, and an unique affine isometry $T$ of $\mathbb{R}^{2}$ for which the curve $T \circ \eta_{a, b}$ possesses the following properties (we omit the dependence of $a, b, T$ on the indices $i, l$ and $n$ ):

$$
\left\{\begin{array}{l}
T \circ \eta_{a, b}(0)=\tilde{c}_{n}^{i, l}\left(\beta_{n}^{i, l}\right) ; \quad T \circ \eta_{a, b}(1)=\tilde{c}_{n}^{i, l+1}\left(\alpha_{n}^{i, l+1}\right) ; \\
\left(T \circ \eta_{a, b}\right)^{\prime}(0)=v_{+}^{i, l} ; \quad\left(T \circ \eta_{a, b}\right)^{\prime}(1)=v_{+}^{i, l} .
\end{array}\right.
$$

One can easily check that

$$
|b| \leqslant 2 \delta_{n}, \quad a \geqslant \frac{1}{2} \bar{s} ; \quad \text { for } n \text { large; }
$$

see Section 2.2 for the definition of $\bar{s}$. From these equations and from (52), it follows that

$$
\left|\frac{\left(T \circ \eta_{a, b}\right)^{\prime}}{\left|\left(T \circ \eta_{a, b}\right)^{\prime}\right|}-v_{i}\right| \leqslant 8 \frac{\delta_{n}}{\bar{s}(P)} ; \quad\left|\kappa\left(T \circ \eta_{a, b}\right)\right| \leqslant 16 \frac{\delta_{n}}{\bar{s}(P)} .
$$

Denote by $\tilde{C}_{n}^{i, l}$ the curve $\eta_{a, b}$, where $a, b$ are chosen as above depending on $i, l, n$, and consider

$$
\tilde{\gamma}_{n}^{i}=\bar{c}_{n}^{1,1} * \tilde{C}_{n}^{1,1} * \bar{c}_{n}^{1,2} * \cdots * \bar{c}_{n}^{1, j_{i}} * \tilde{C}_{n}^{1, j_{i}} .
$$

It follows from the first equation in (53) that if $M$ is sufficiently large, then the curves $\tilde{\gamma}_{n}^{i}, i=$ $1, \ldots, k$ are simple, mutually disjoint, and the union of their images is the boundary of a piecewise $C^{2}$ set $\tilde{E}_{n} \subseteq \mathbb{R}^{2}$. It is clear that $\tilde{E}_{n} \rightarrow P$ in $L^{1}\left(\mathbb{R}^{2}\right)$. 
Let $\varepsilon_{n} \rightarrow 0$ : for every $i \in\{1, \ldots, k\}$ and every $l \in\left\{1, \ldots, j_{i}\right\}$, let $a^{i, l}, b^{i, l}$, etc., be the analogous of $a, b, c, d$ in the proof of Proposition 4.1 when we consider $v^{i, l}, v_{-}^{i, l}$ and $v_{+}^{i, l}$. Since $\varphi$ is assumed to be of class $C^{1}$, we can choose $\delta_{n} \rightarrow 0$ and $e^{i, l}(n), f^{i, l}(n)$ with the following properties:

(i) $\lim _{n} \frac{\delta_{n}}{\varepsilon_{n}\left(\left|e^{i, l}(n)\right|+\mid f^{i, l}(n)\right) \mid}=+\infty \quad$ for all $i \in\{1, \ldots, k\}$ and every $l \in\left\{1, \ldots, j_{i}\right\}$;

(ii) $\lim _{n} \frac{1}{\varepsilon_{n}} C\left(\frac{8}{\bar{s}} \delta_{n}\right)=0$;

see (27) for the definition of $C(\delta)$.

We have

$$
\int_{[0,1]} \frac{1}{\varepsilon_{n}} \varphi\left(\frac{\left(\tilde{C}_{n}^{i, l}\right)^{\prime}}{\left|\left(\tilde{C}_{n}^{i, l}\right)^{\prime}\right|}\right) \mathrm{d} t+\varepsilon_{n} \int_{[0,1]} \kappa^{2}\left(\tilde{C}_{n}^{i, l}\right) \mathrm{d} t \leqslant \frac{1}{\varepsilon_{n}} C\left(\frac{8}{\bar{s}} \delta_{n}\right)+\varepsilon_{n}\left(\frac{16}{\bar{s}}\right)^{2} \delta_{n}^{2} .
$$

From property (ii) above and from (53), it follows that

$$
\lim _{n}\left(\int_{[0,1]} \frac{1}{\varepsilon_{n}} \varphi\left(\frac{\left(\tilde{C}_{n}^{i, l}\right)^{\prime}}{\left|\left(\tilde{C}_{n}^{i, l}\right)^{\prime}\right|}\right) \mathrm{d} t+\varepsilon_{n} \int_{[0,1]} \kappa^{2}\left(\tilde{C}_{n}^{i, l}\right) d t\right)=0
$$

By Remark 4.1, for every $i \in\{1, \ldots, k\}$, every $l \in\left\{1, \ldots, j_{i}\right\}$ and every $n$ sufficiently large it is possible to choose a curve $\bar{C}_{n}^{i, l}:\left[\alpha_{n}^{i, l}, \beta_{n}^{i, l}\right] \rightarrow \mathbb{R}^{2}$ such that

$$
\begin{gathered}
\left|\bar{C}_{n}^{i, l}(t)-\bar{c}_{n}^{i, l}(t)\right| \leqslant 2 \varepsilon_{n}\left(\left|e^{i, l}(n)\right|+\left|f^{i, l}(n)\right|\right) ; \\
\bar{C}_{n}^{i, l} \text { coincides with } \bar{c}_{n}^{i, l} \text { in a neighbourhood of }\left\{\alpha_{n}^{i, l}, \beta_{n}^{i, l}\right\} ; \\
\int_{\left[\alpha_{n}^{i, l}, \beta_{n}^{i, l}\right]} \frac{1}{\varepsilon_{n}} \varphi\left(\frac{\left(\bar{C}_{n}^{i, l}\right)^{\prime}}{\left|\left(\bar{C}_{n}^{i, l}\right)^{\prime}\right|}\right) \mathrm{d} t+\int_{\left[\alpha_{n}^{i, l}, \beta_{n}^{i, l}\right]} \varepsilon_{n} \kappa^{2}\left(\bar{C}_{n}^{i, l}\right) \mathrm{d} t \rightarrow g\left(\nu_{-}^{i, l}, \nu_{+}^{i, l}\right) .
\end{gathered}
$$

Let $\bar{\gamma}^{i}$ be the curve defined by

$$
\tilde{\gamma}_{n}^{i}=\bar{C}_{n}^{i, 1} * \tilde{C}_{n}^{i, 1} * \bar{C}_{n}^{i, 2} * \cdots * \bar{C}_{n}^{i, j_{i}} * \tilde{C}_{n}^{i, j_{i}} .
$$

From (56) it follows that the curve $\tilde{\gamma}_{n}^{i}, i=1, \ldots, k$, are curves of class $C^{2}$, while (55) implies that they are simple, mutually disjoint, and the union of their images is the boundary of a $C^{2}$ set $\bar{E}_{n} \subseteq \mathbb{R}^{2}$. Again, $\tilde{E}_{n} \rightarrow P$ in $L^{1}\left(\mathbb{R}^{2}\right)$. Moreover from (57) one can deduce that

$$
\lim \sup _{n} F_{\varepsilon_{n}}\left(\bar{E}_{n}\right) \leqslant \sum_{v \in V(P)} E(v) .
$$

This concludes the proof. 


\subsection{A symmetric case}

In this section we treat the case in which the admissible polygons are polyrectangles, and the function $\varphi$ is symmetric with respect to the axes $x$ and $y$. A direct proof of Theorem 5.1 is also presented in [10], Appendix B.

THEOREM 5.1 Let $\mathbf{e}_{1}, \mathbf{e}_{2}$ be the canonical basis of $\mathbb{R}^{2}$, and suppose that $\varphi$ satisfies the conditions

$$
\varphi^{-1}(0)=\left\{v_{1}, \ldots, v_{4}\right\}, \quad \text { where } \quad v_{1}=\mathbf{e}_{1}, \quad v_{2}=\mathbf{e}_{2}, \quad v_{3}=-\mathbf{e}_{1}, \quad v_{4}=-\mathbf{e}_{2},
$$

and

$$
g_{0}:=g\left(v_{i}, v_{i+1}\right) \quad \text { is independent of } i=1, \ldots, 4 .
$$

Then the admissible polygons are polyrectangles, and for every $P \in \mathcal{P}$

$$
G(P)=g_{0} \times \#\{\text { vertices of } P\} .
$$

Proof. Let us prove first the $\Gamma$-lim inf inequality. We note that if $P \in \mathcal{R}$, then one has

$$
F_{\mathcal{R}}(P)=\#\{\text { vertices of } P\}=\#\{\text { sides of } P\} .
$$

Let $E \in \mathcal{P}$, and let $E_{k} \in \mathcal{R}, E_{k} \rightarrow E$ in $L^{1}\left(\mathbb{R}^{2}\right)$. Then, since it must be \#\{sides of $\left.E_{k}\right\} \geqslant$ \#\{sides of $E$ \} for $k$ large, it follows from (60) that

$$
F_{\mathcal{R}}\left(E_{k}\right) \geqslant \#\{\text { sides of } E\} \geqslant \#\{\text { vertices of } E\}, \quad \text { for } k \text { large. }
$$

Hence we have also

$$
G(E)=s c^{-}\left(F_{\mathcal{R}}\right)(E) \geqslant \#\{\text { vertices of } E\},
$$

which is the $\Gamma$-lim inf inequality. Let us prove now the $\Gamma$-lim sup inequality. Given a polyrectangle $E$, and given a number $\sigma>0$, consider the set $E_{\sigma}$ defined by

$$
E_{\sigma}=\{x \in E: \operatorname{dist}(x, \partial E) \leqslant \sigma\} .
$$

Then, if $\sigma$ is sufficiently small, $E_{\sigma} \in \mathcal{R}$, and \#\{sides of $\left.E_{\sigma}\right\} \leqslant \#\{$ sides of $E\}$. This concludes the proof.

\section{Pathological cases}

In this section we consider the case in which the uniform boundedness of the perimeter is not required in the definition of convergence. In this situation, it is possible to have the convergence in the $L_{\text {loc }}^{1}\left(\mathbb{R}^{2}\right)$ sense without having convergence in $L^{1}\left(\mathbb{R}^{2}\right)$, so we are led to defining

$$
\bar{G}(E)=\inf \left\{\liminf _{n} F_{\varepsilon_{n}}\left(E_{n}\right): E_{n} \rightarrow E \text { in } L_{\text {loc }}^{1}\left(\mathbb{R}^{2}\right)\right\} .
$$

We recall that, by Theorem 2.1, G(E) $=\inf \left\{\liminf _{n} F_{\varepsilon_{n}}\left(E_{n}\right): E_{n} \rightarrow E\right.$ in $L^{1}\left(\mathbb{R}^{2}\right), \sup _{n} \mathcal{H}_{1}$ $\left(\partial E_{n}\right)<+\infty$ \}, so it is clearly $\bar{G}(E) \leqslant G(E)$. In Section 6.1 we describe the asymptotic shape of the subsequences $\left(E_{n}\right)$ for which $\sup _{n} F_{\varepsilon_{n}}\left(E_{n}\right)<+\infty$, highlighting similarities with Section 3 . However, in general $\bar{G}<G$. In Section 6.2 we are able to exhibit a function $\varphi$ and a polygon $P$ for which $\bar{G}(P)$ is strictly less than $G(P)$. 


\subsection{Asymptotic shape of minimizers}

In this section we describe the limit shape of a sequence of sets $\left(E_{n}\right)$ for which just condition $\left(H_{3}\right)$ holds, while condition $\left(\mathrm{H}_{2}\right)$ — the uniform boundedness of the perimeter-is lifted.

We suppose that $\partial E_{n}$ possesses just one connected component; the general case requires only simple modifications. Let $\gamma_{n}$ be a parametrization of $\partial E_{n}$ proportional to the arc length. First, we note that Lemmas 3.1 and 3.2 remain unchanged, so we can define the quantities $\delta_{n} \rightarrow 0, I_{n}$ and $J_{n}$ with $\left|I_{n}\right| \rightarrow 0$, just as we did in Section 3. In general, we do not have uniform convergence on the components of $J_{n}$ as in Lemma 3.3. However, it can be recovered under a suitable rescaling.

LEMMA 6.1 Let $J_{n}$ be defined as in (30), and let $\left(\theta_{n}^{h}, \sigma_{n}^{h+1}\right)$ be a component of $J_{n}$ such that

$$
\dot{\gamma}_{n}\left(\theta_{n}^{h}\right)=\left|\dot{\gamma}_{n}\right|\left(v_{i} \pm \delta_{n}\right) \quad \text { for some } v_{i} \in\left\{v_{1}, \ldots, v_{N}\right\}
$$

and such that $\left|\gamma_{n}\left(\theta_{n}^{h}\right)-\gamma_{n}\left(\sigma_{n}^{h+1}\right)\right| \rightarrow+\infty$ as $n \rightarrow+\infty$. Let $\tilde{\gamma}_{n}:\left(\theta_{n}^{h}, \sigma_{n}^{h+1}\right)$ be defined by

$$
\tilde{\gamma}_{n}(t)=\frac{1}{\left|\gamma_{n}\left(\theta_{n}^{h}\right)-\gamma_{n}\left(\sigma_{n}^{h+1}\right)\right|}\left(\gamma_{n}(t)-\gamma_{n}\left(\theta_{n}^{h}\right)\right) .
$$

Then we have

$$
\sup _{t \in\left(\theta_{n}^{h}, \sigma_{n}^{h+1}\right)}\left|\tilde{\gamma}_{n}(t)-v_{i} t\right| \rightarrow 0, \quad \text { as } n \rightarrow+\infty .
$$

Proof. We have $\left|\tilde{\gamma}_{n}(t)\right| \leqslant C$ on $\left(\theta_{n}^{h}, \sigma^{h+1}\right)$, and moreover

$$
\int_{\left(\theta_{n}^{h}, \sigma_{n}^{h+1}\right)} \frac{1}{\varepsilon_{n}} \varphi\left(\frac{\dot{\tilde{\gamma}}_{n}}{\left|\dot{\tilde{\gamma}}_{n}\right|}\right) \mathrm{d} t \leqslant \int_{\left(\theta_{n}^{h}, \sigma_{n}^{h+1}\right)} \frac{1}{\varepsilon_{n}} \varphi\left(\frac{\dot{\gamma}_{n}}{\left|\dot{\gamma}_{n}\right|}\right) \mathrm{d} t .
$$

Hence, considering the curve $\tilde{\gamma}_{n}$, we are in the same situation of Lemma 3.3, so our statement follows.

Passing to a subsequence, we find an integer $k$, and $k$ sequences of points $\left(x_{n}^{1}\right), \ldots,\left(x_{n}^{k}\right)$ such that

$$
\operatorname{dist}\left(\gamma_{n}\left(I_{n}\right),\left\{x_{n}^{1}, \ldots, x_{n}^{k}\right\}\right) \rightarrow 0, \quad \text { as } n \rightarrow+\infty
$$

In this case, the mutual distances between the points $x_{n}^{i}$ can diverge. However, it turns out that the sequences of points $\left\{x_{n}^{1}, \ldots, x_{n}^{k}\right\}$ arrange in 'clusters', and the limit shape of some rescaled portion of $E_{n}$ is still polygonal.

In fact, let

$$
d_{n}^{1}=\sup \left\{\left|x_{n}^{i}-x_{n}^{j}\right|: i, j \in\{1, \ldots, k\}, i \neq j\right\},
$$

and consider the sequence of sets

$$
E_{n}^{1}=\left(d_{n}^{1}\right)^{-1}\left(E_{n}-x_{n}^{1}\right)
$$

Let $\gamma_{n}^{1}$ be a parametrization of $\partial E_{n}^{1}$. Then, there exists a number $k^{1} \leqslant k$ and $k_{1}$ sequences of points $\left(x_{n}^{1,1}\right), \ldots,\left(x_{n}^{1, k_{1}}\right)$ such that

$$
\operatorname{dist}\left(\gamma_{n}^{1}\left(I_{n}\right),\left\{x_{n}^{1,1}, \ldots, x_{n}^{1, k_{1}}\right\}\right) \rightarrow 0, \quad \text { as } n \rightarrow+\infty .
$$


From Lemma 6.1, it is easy to see that the sequence $E_{n}^{1}$ converges in $L^{1}\left(\mathbb{R}^{2}\right)$ to some admissible polygon $P^{1} \in \mathcal{P}$.

If we choose a different rescaling for the set $E_{n}$, we can obtain some 'finer' structures of these sets. In fact, consider the set of indices $\left\{i_{1}, \ldots, i_{j}\right\} \subseteq\{1, \ldots, k\}$, for which

$$
\lim _{n}\left(d_{n}^{1}\right)^{-1}\left|x_{n}^{i_{l}}-x_{n}^{1}\right| \rightarrow 0, \quad l=1, \ldots, j
$$

and define $d_{n}^{2}$ to be

$$
d_{n}^{2}=\sup \left\{\left|x_{n}^{i_{l}}-x_{n}^{i_{h}}\right|: l, h \in\left\{i_{1}, \ldots, i_{j}\right\}, l \neq h\right\} ;
$$

it is clear that $\left(d_{n}^{1}\right)^{-1} d_{n}^{2} \rightarrow 0$. Consider the sequence of sets $E_{n}^{2}$

$$
E_{n}^{2}=\left(d_{n}^{2}\right)^{-1}\left(E_{n}-x_{n}^{1}\right)
$$

Then, using the arguments above, one can check that $E_{n}^{2} \rightarrow P^{2}$ in $L_{\text {loc }}^{1}\left(\mathbb{R}^{2}\right)$, where $P^{2} \subseteq \mathbb{R}^{2}$ is a set whose boundary is composed of segments, half-lines or lines oriented in the directions $\left\{v_{1}, \ldots, v_{N}\right\}$. In some sense, $P^{2}$ could be considered as a polygon with some sides of infinite length.

Of course, the same result holds true if one considers suitable rescalings at the points $x_{n}^{i}$ for $i \neq 1$.

\subsection{An example in which $\overline{\mathbf{G}} \neq \mathbf{G}$}

In this section we consider the following particular case, namely $\varphi^{-1}(0)=\left\{v_{1}, \ldots, v_{5}\right\}$ with

$$
v_{1}=(1,0) ; \quad v_{2}=(0,1) ; \quad v_{3}=\frac{\sqrt{2}}{2}(-1,1) ; \quad v_{4}=\frac{\sqrt{2}}{2}(-1,-1) ; \quad v_{5}=(0,-1),
$$

and

$$
g\left(v_{1}, v_{2}\right)=g\left(v_{2}, v_{3}\right)=g\left(v_{4}, v_{5}\right)=g\left(v_{1}, v_{2}\right)=1 ; \quad g\left(v_{5}, v_{1}\right)=5
$$

Let $p_{i}, q_{i} \in \mathbb{R}^{2}, i=1, \ldots, 3$, be given by

$$
p_{1}=(0,0), \quad p_{2}=(1,0), \quad p_{3}=(1,1) ; \quad q_{1}=(2,0), \quad q_{2}=(3,0), \quad q_{3}=(2,1),
$$

and let $P$ be the polygon defined as follows (see Fig. 3(a)):

$$
P=\left\{\sum_{i=1}^{3} t_{i} p_{i} \mid t_{i} \geqslant 0, \sum_{i=1}^{3} t_{i}=1\right\} \cup\left\{\sum_{i=1}^{3} t_{i} q_{i} \mid t_{i} \geqslant 0, \sum_{i=1}^{3} t_{i}=1\right\} .
$$

It is clear from (61) that $P \in \mathcal{P}$. We show that in this case $\bar{G}(P)$ is strictly less than $G(P)$.

In fact, let $\left(E_{n}\right) \subseteq \mathcal{R}$ be a sequence of sets of class $C^{2}$ as in Fig. 3(b). It is clear that the boundary of $E_{n}$ has just one component and from (62) one can check that $F_{\mathcal{R}}\left(P_{n}\right)=17+o(1)$, where $o(1) \rightarrow 0$ as $n \rightarrow+\infty$.

Now, suppose by contradiction that $G(P)=\bar{G}(P) \leqslant 17$, namely that there exists $\left(E_{n}\right) \subseteq \mathbb{R}^{2}$ with

$$
E_{n} \rightarrow E \text { in } L^{1}\left(\mathbb{R}^{2}\right), \quad \sup _{n} \mathcal{H}_{1}\left(\partial E_{n}\right)<+\infty, \quad \lim _{n} F_{\varepsilon_{n}}\left(E_{n}\right) \leqslant 17
$$




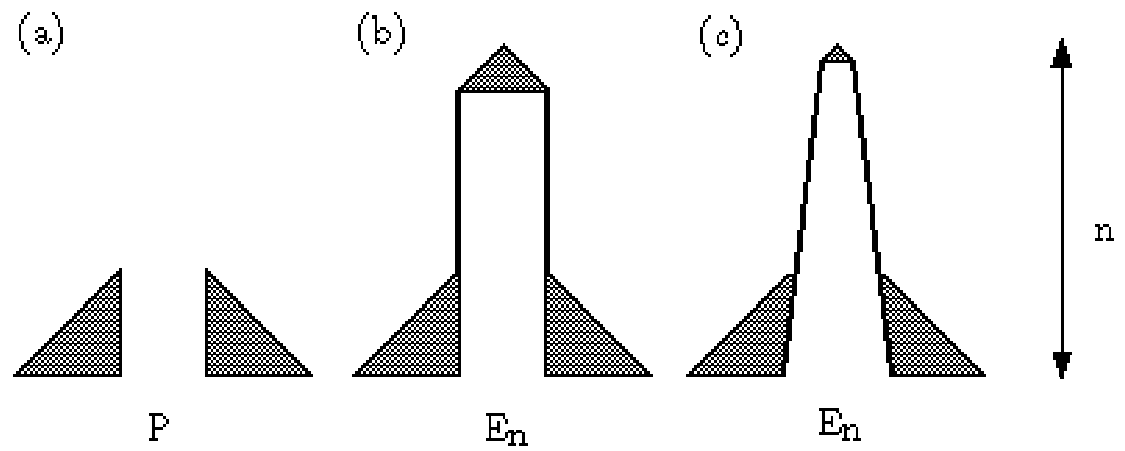

FIG. 3. A set with $\bar{G}(P)<G(P)$ and its optimal approximations

Passing to a subsequence, we can assume that the number of the components of $\partial E_{n}$ is a fixed number $k$ independent of $n$. By Lemma 3.1, it turns out that $F_{\varepsilon_{n}}\left(E_{n}\right) \geqslant 9 k+o(1)$, so, since we are assuming that $F_{\varepsilon_{n}}\left(E_{n}\right) \leqslant 17+o(1)$, it follows that $k=1$.

Let $\gamma_{n}: S^{1} \rightarrow \mathbb{R}^{2}$ be a parametrization of $\partial E_{n}$ proportional to the arc length. Then we can apply Proposition 3.2, and we find a curve $\gamma: S^{1} \rightarrow \mathbb{R}^{2}, \gamma \in \mathcal{C}$, for which $\gamma_{n} \rightarrow \gamma$ uniformly on $S^{1}$, and for which $P=\left\{x \in \mathbb{R}^{2}: \operatorname{ind}(\gamma, x)=1\right\}$.

Consider the set

$$
A=\left\{t \in S^{1}: 1<(\gamma)_{x}(t)<2, \dot{\gamma}(t) \in\left\{v_{3}, v_{4}\right\}\right\} .
$$

Since $\gamma$ has just one component, it must be $A \neq \emptyset$, and since $-v_{3}$ and $-v_{4}$ do not belong to $\varphi^{-1}(0)$, it should be $\gamma(A) \subseteq \partial P$, which is a contradiction.

REMARK 6.1 It is possible to have $\bar{G}(P)<G(P)$ also when the (strong) $L^{1}$ convergence is required in the definition of $\bar{G}$. In fact, if $\varphi$ is of class $C^{1}$, one could choose a sequence of approximating sets $\left(E_{n}\right)$ as in Fig. 3(c). Reasoning as in Section 5, one can prove that $F_{\varepsilon_{n}}\left(E_{n}\right)=$ $17+o(1)$.

\section{Acknowledgements}

AB is part of the MURST project Calculus of Variations; AM has been supported by MURST under the project Variational Methods and Nonlinear Differential Equations and by a Fulbright Fellowship for the academic year 2000-2001. We thank Antonio Di Carlo for a useful critical reading of the manuscript. A great part of the present work was accomplished at SISSA, Trieste.

\section{REFERENCES}

1. Alberti, G. \& Bellettini, G. A non-local anisotropic model for phase transitions: asymptotic behaviour of rescaled energies. European J. Appl. Math. 9, (1998) 261-284.

2. Alberti, G. \& Bellettini, G. A nonlocal anisotropic model for phase transitions. I. The optimal profile problem. Math. Ann. 310, (1998) 527-560.

3. Alberti, G., Bellettini, G., Cassandro, M., \& Presutti, E. Surface tension in Ising systems with Kac potentials. J. Statist. Phys. 82, (1996) 743-796. 
4. Ambrosio, L. \& Braides, A. Functionals defined on partitions in sets of finite perimeter. II. Semicontinuity, relaxation and homogenization. J. Math. Pures Appl. 69, (1990) 307-333.

5. Ambrosio, L., Fusco, N., \& Pallara, D. Functions of Bounded Variation and Free Discontinuity Problems. Oxford University Press, Oxford (2000).

6. Bellettini, G., Dal Maso, G., \& Paolini, M. Semicontinuity and relaxation properties of a curvature depending functional in 2D. Ann. Scuola Norm. Sup. Pisa Cl. Sci. 20, (1993) 247-297.

7. Bellettini, G., Goglione, R., \& Novaga, M. Approximation to driven motion by crystalline curvature in two dimensions. Adv. Math. Sci. Appl. 10, (2000) 467-493.

8. Bodineau, T. The Wulff construction in three and more dimensions. Comm. Math. Phys. 207, (1999) 197-229.

9. Bodineau, T., IOFFE, D., \& VELENIK, Y. Rigorous probabilistic analysis of equilibrium crystal shapes. Probabilistic techniques in equilibrium and nonequilibrium statistical physics. J. Math. Phys. 41, (2000) 1033-1098.

10. BRAIDES, A. Approximation of Free-Discontinuity Problems. Springer, Berlin (1998).

11. BRAides, A. $\Gamma$-convergence for Beginners. Oxford University Press, Oxford (2002).

12. Coscia, A. On curvature sensitive image segmentation. Nonlinear Anal. 39, (2000) 711-730.

13. Dal Maso, G. An Introduction to $\Gamma$-convergence. Progress in Nonlinear Differential Equations and their Applications 8. Birkhäuser, Boston (1993).

14. Dal Maso, G. \& Romanelli, M. Paper in preparation.

15. FonseCA, I. Variational methods for elastic crystals. Arch. Rational Mech. Anal. 97, (1987) 189-220.

16. FonseCA, I. The lower quasiconvex envelope of the stored energy function for an elastic crystal. J. Math. Pures Appl. 67, (1988) 175-195.

17. Giga, Y., Gurtin, M. E., \& Matias, J. On the dynamics of crystalline motions. Japan J. Indust. Appl. Math. 15, (1998) 7-50.

18. Giusti, E. Minimal Surfaces and Functions of Bounded Variation. Monographs in Mathematics 80. Birkhäuser, Boston, MA (1984).

19. Goglione, R. \& PAOLini, M. Numerical simulations of crystalline motion by mean curvature with Allen-Cahn relaxation. Free Boundary Problems, Theory and Applications (Zakopane, 1995), Pitman Res. Notes Math. Ser. vol. 363. Longman, Harlow (1996) pp. 203-216.

20. Gurtin, M. E. Some results and conjectures in the gradient theory of phase transitions, in "Metastability and incompletely posed problems, Proc. Workshop, Minneapolis/Minn. 1984/85". IMA Vol. Math. Appl. 3, (1987) 135-146.

21. Gurtin, M. E., Soner, H. M., \& Souganidis, P. E. Anisotropic motion of an interface relaxed by the formation of infinitesimal wrinkles. J. Differential Equations 119, (1995) 54-108.

22. Luckhaus, S. \& ModicA, L. The Gibbs-Thompson relation within the gradient theory of phase transitions. Arch. Rational Mech. Anal. 107, (1989) 71-83.

23. MantegazZa, C. Curvature varifolds with boundary. J. Differential Geom. 43, (1996) 807-843.

24. ModicA, L. The gradient theory of phase transitions and the minimal interface criterion. Arch. Rational Mech. Anal. 98, (1987) 123-142.

25. ModicA, L. \& Mortola, S. Un esempio di $\Gamma^{-}$-convergenza. Boll. Un. Mat. Ital. B 14, (1977) 285299.

26. Mumford, D. Elastica and computer vision. Algebraic Geometry and its Applications (West Lafayette, IN, 1990). Springer, New York (1994) pp. 491-506.

27. Nitzberg, M., Mumford, D., \& Shiota, T. Filtering, Segmentation and Depth. Lecture Notes in Computer Science, 662. Springer, Berlin (1993).

28. TAYLOR, J. E. On the global structure of crystalline surfaces. Discrete Comput. Geom. 6, (1991) 225-262.

29. TAYLOR, J. E. Constructions and conjectures in crystalline nondifferential geometry. Differential Geometry, Pitman Monographs Surveys Pure Appl. Math. vol. 52. Longman, Harlow (1991) pp. 321-336.

30. TAYlor, J. E. \& CAHn, J. W. Diffuse interfaces with sharp corners and facets: phase field models with strongly anisotropic surfaces. With an appendix by Jason Yunger. Phys. D 112, (1998) 381-411. 